\title{
On the oscillator realization of conformal $U(2,2)$ quantum particles and their particle-hole coherent states
}

\author{
M. Calixto and E. Pérez-Romero \\ Departamento de Matemática Aplicada, Facultad de Ciencias, Campus de Fuentenueva, \\ 18071 Granada, Spain
}

\begin{abstract}
We revise the unireps. of $U(2,2)$ describing conformal particles with continuous mass spectrum from a many-body perspective, which shows massive conformal particles as compounds of two correlated massless particles. The statistics of the compound (boson/fermion) depends on the helicity $h$ of the massless components (integer/half-integer). Coherent states (CS) of particle-hole pairs ("excitons") are also explicitly constructed as the exponential action of exciton (non-canonical) creation operators on the ground state of unpaired particles. These CS are labeled by points $Z(2 \times 2$ complex matrices $)$ on the Cartan-Bergman domain $\mathbb{D}_{4}=U(2,2) / U(2)^{2}$, and constitute a generalized (matrix) version of Perelomov $U(1,1)$ coherent states labeled by points $z$ on the unit disk $\mathbb{D}_{1}=U(1,1) / U(1)^{2}$. Firstly we follow a geometric approach to the construction of CS, orthonormal basis, $U(2,2)$ generators and their matrix elements and symbols in the reproducing kernel Hilbert space $\mathcal{H}_{\lambda}\left(\mathbb{D}_{4}\right)$ of analytic square-integrable holomorphic functions on $\mathbb{D}_{4}$, which carries a unitary irreducible representation of $U(2,2)$ with index $\lambda \in \mathbb{N}$ (the conformal or scale dimension). Then we introduce a many-body representation of the previous construction through an oscillator realization of the $U(2,2)$ Lie algebra generators in terms of eight boson operators with constraints. This particle picture allows us for a physical interpretation of our abstract mathematical construction in the many-body jargon. In particular, the index $\lambda$ is related to the number $2(\lambda-2)$ of unpaired quanta and to the helicity $h=(\lambda-2) / 2$ of each massless particle forming the massive compound.
\end{abstract}

PACS: 03.65.Fd, 11.25.Hf, 03.65.Ge, 02.40.Tt, 71.35.Lk,

MSC: 81R30, 81R05, 81R25, 81S10, 32Q15

Keywords: Conformal group, coherent states, oscillator realization, particle-hole excitations, twistors. 


\section{Introduction}

The generalization of Poincaré symmetry of space-time to conformal symmetry is a recurrent subject with a vast literature in mathematical and particle physics. However, its physical interpretation and its broken character keep raising controversy. Special conformal transformations can be either interpreted as transitions to systems of relativistic, uniformly accelerated observers (see e.g. Refs.[1, 2, 3]), the temporal component being a kind of kinematical red-shift (resembling Hubble's law) [4], or related to the Weyl's idea of different lengths in different points of space time [5], or to the Kastrup's interpretation as geometrical gauge transformations of the Minkowski space [6], etc. Moreover, it is usually assumed that exact scale invariance is physically unacceptable since it implies that the mass spectrum is either continuous or all masses are zero.

In this article we revisit the conformal group $S U(2,2)$ and some of its positive mass unirreps (discrete series, to be more precise) from an oscillator realization that provides an interesting many-body interpretation. Since Jordan [7] (see also [8]) and Schwinger [9] introduced a way of representing the matrix generators of a certain symmetry in terms of bilinear products (field operators) of either boson or fermion type, this oscillator representation (also called Jordan-Schwinger mapping) has been widely used to provide a treatment of representations of Lie groups, with the calculation of matrix elements of finite and infinitesimal group transformations in the bases of coherent and Fock states; see, e.g., Biedenharn \& Louck [8] for unitary groups, Moshinsky et al. [10, 11, 12, 13] and Iachello et al. [14, 15, 16] in the context of molecular, atomic and nuclear physics, and [17, 18, 19] for the case of Cayley-Klein groups. Many-body quantum systems in the second quantized field formalism fit well into this picture, where Hamiltonians (and other relevant operators) are multidimensional quadratic in boson or fermion creation and annihilation operators.

For the conformal group $S U(2,2)$, the oscillator realization of the so called ladder representations, describing massless particles, where studied long time ago by [20, 21]. This Jordan-Schwinger-like mapping is in fact extensible to general pseudo-unitary grups $U(p, q)$ [22, 23] and it became popular after [24], who discussed the use of $U(6,6)$ to classify hadrons; in this case barions and antibarions belong to mutually conjugate representations with respect to $U(6)$. The case of positive-mass unireps. of $U(2,2)$ was already discussed in, for example, [25, 26, 27], and more recently by us in [28, 29, 30], but their oscillator realizations have not been studied as thoroughly as the simpler case of ladder (most degenerate) representations. An inspiring article on this subject has been recently published in [31], in the context of deformation quantization. Here we further develop this oscillator realization of discrete series representations of $S U(2,2)$ by providing explicit expressions for orthonormal basis vectors, coherent states (see standard references and textbooks [32, 33, 34, 35, 36] on this subject), operators and their matrix elements and symbols. As far as we know, explicit expressions of this kind have not been written before and we think that they will be useful not only for the better understanding of the structure of conformal quantum particles but also in some condensed matter applications

related to pairing (particle-hole) models (see later in Section 6 for a physical interpretation 
of our construction in this sense).

The oscillator realization of $U(2,2)$ fits well into the twistor program introduced by $\mathrm{R}$. Penrose and coworkers in the 1960's [37, 38, 39, 40, 41] as an approach to the unification of quantum theory with gravity. These constructions had wide application across pure and applied mathematics, but not so extensive in basic physics, mainly because twistor space is chiral and treats the left and right handed parts of physical fields differently. However, Witten's paper [42] on twistorial representations of scattering amplitudes showed how to overcome this, and left-right symmetric theories on space-time naturally arise when string theory is introduced into twistor space. This is the subject of much recent activity, with a reworking on the theory of particle interactions in twistor language (see e.g. [43] and references therein). We comment in Sec. 6 on this twistor picture and its relation to the oscillator realization by making use of nonlinear sigma-model Lagrangians on cosets $\mathbb{D}_{3}=U(2,2) / U(2,1) \times U(1)$ (for massless particles) and $\mathbb{D}_{4}=U(2,2) / U(2)^{2}$ (for massive particles), the latter being related to the forward tube domain of the complex Minkowski space.

The paper is organized as follows. In Section 2 we briefly remind the Lie algebra structure and coordinate systems of $U(2,2)$ adapted to the fibration $U(2)^{2} \rightarrow U(2,2) \rightarrow \mathbb{D}_{4}$. In Section 3 we construct a coherent state (CS) system labeled by points of $\mathbb{D}_{4}$ in the (reproducing kernel) Hilbert space $\mathcal{H}_{\lambda}\left(\mathbb{D}_{4}\right)$ of analytic square-integrable holomorphic functions on $\mathbb{D}_{4}$ with a given measure. This corresponds to a given square-integrable irreducible representation of $U(2,2)$ with positive integer index $\lambda$ representing the conformal or scale dimension. Firstly we follow a geometric approach to the construction of CS on $\mathbb{D}_{4}$, in part inspired by the method of orbits in geometric quantization due to Kirillov-Kostant-Souriau [44, 45, 46] and the Borel-Weil-Bott theorem [47], which relate quantization, geometry and the representation theory for classical groups. In Section 4 we explicitly compute the infinitesimal generators (Poincaré plus dilations and special conformal transformations) of the representation of $U(2,2)$ on $\mathcal{H}_{\lambda}\left(\mathbb{D}_{4}\right)$, their matrix elements in an orthonormal basis and their operator symbols in coherent states. Then in Section 5 we introduce an oscillator realization of the $u(2,2)$ Lie algebra in terms of eight boson creation, $a_{\mu}^{\dagger}, b_{\mu}^{\dagger}$, and annihilation, $a_{\mu}, b_{\mu}, \mu=0,1,2,3$, operators, and express the orthonormal basis of $\mathcal{H}_{\lambda}\left(\mathbb{D}_{4}\right)$ in terms of the Fock basis with constraints on the occupancy numbers. An expression of $\mathbb{D}_{4}$ CS as Bose-Einstein-like condensates of excitons is also provided. This way we connect the abstract construction of Section 3 with the "many-body picture". This realization differs from the standard boson representation of $u(2,2)$ in terms of four bosons, leading to ladder representations for massless particles and related to the pseudo complex projective $\mathbb{D}_{3}$. Actually, conformal massive particles turn out to be a compound of two massless correlated particles, something that might result strange in principle. The compound wavefunctions are symmetric (resp. antisymmetric) under the interchange of the two massless constituents for $\lambda$ even (resp. odd), the helicity of each massless particle being $(\lambda-2) / 2$. The $0+1$ dimensional case, which is described by Perelomov su $(1,1)$ CS on the unit disk $\mathbb{D}_{1}=U(1,1) / U(1)^{2}$, is treated in parallel all along the paper, to better appreciate the role played by spin and to stress the similarities and differences between $\mathbb{D}_{4}$ and $\mathbb{D}_{1} \mathrm{CS}$, the former being a generalized (matrix) version of the latter. Section 6 is de- 
voted to some comments on the Lagrangian picture and possible physical interpretations, not only for the better understanding of the structure of conformal quantum particles, but also for possible applications in general pairing systems like nuclear pairing phenomenon, superconductivity in solid state physics, Bose-Einstein condensation of excitons, etc.

\section{$2 U(2,2)$ coordinate systems and generators}

The usual action of the conformal group $S O(4,2)$ on Minkowski spacetime $\mathbb{R}^{4} \ni x^{\mu}$ is comprised of Poincaré spacetime translations $x^{\prime \mu}=x^{\mu}+b^{\mu}$ and Lorentz transformations $x^{\prime \mu}=\Lambda_{\nu}^{\mu} x^{\nu}$ augmented by dilations $x^{\prime \mu}=\rho x^{\mu}$ and relativistic uniform accelerations (special conformal transformations) $x^{\prime \mu}=\frac{x^{\mu}+a^{\mu} x^{2}}{1+2 a x+a^{2} x^{2}}$. We shall denote by $P^{\mu}, M^{\mu \nu}, D$ and $K^{\mu}$ the corresponding Lie algebra generators, respectively. Here we are interested in the usual $4 \times 4$ matrix realization of these conformal Lie algebra generators

$$
\begin{aligned}
D & =\frac{\gamma^{5}}{2}, M^{\mu \nu}=\frac{\left[\gamma^{\mu}, \gamma^{\nu}\right]}{4}=\frac{1}{4}\left(\begin{array}{cc}
\sigma^{\mu} \check{\sigma}^{\nu}-\sigma^{\nu} \check{\sigma}^{\mu} & 0 \\
0 & \check{\sigma}^{\mu} \sigma^{\nu}-\check{\sigma}^{\nu} \sigma^{\mu}
\end{array}\right), \\
P^{\mu} & =\gamma^{\mu} \frac{1+\gamma^{5}}{2}=\left(\begin{array}{cc}
0 & \sigma^{\mu} \\
0 & 0
\end{array}\right), K^{\mu}=\gamma^{\mu} \frac{1-\gamma^{5}}{2}=\left(\begin{array}{cc}
0 & 0 \\
\check{\sigma}^{\mu} & 0
\end{array}\right)
\end{aligned}
$$

in terms of gamma matrices $\gamma^{\mu}$ in, for instance, the Weyl basis

$$
\gamma^{\mu}=\left(\begin{array}{cc}
0 & \sigma^{\mu} \\
\check{\sigma}^{\mu} & 0
\end{array}\right), \gamma^{5}=i \gamma^{0} \gamma^{1} \gamma^{2} \gamma^{3}=\left(\begin{array}{cc}
-\sigma^{0} & 0 \\
0 & \sigma^{0}
\end{array}\right)
$$

where $\check{\sigma}^{\mu} \equiv \sigma_{\mu}=\eta_{\mu \nu} \sigma^{\nu}$ [we are using the convention $\eta=\operatorname{diag}(1,-1,-1,-1)$ for the Minkowski metric] and $\sigma^{\mu}$ are the Pauli matrices (plus identity $\sigma^{0}$ )

$$
\sigma^{0}=\left(\begin{array}{ll}
1 & 0 \\
0 & 1
\end{array}\right), \sigma^{1}=\left(\begin{array}{ll}
0 & 1 \\
1 & 0
\end{array}\right), \sigma^{2}=\left(\begin{array}{cc}
0 & -i \\
i & 0
\end{array}\right), \sigma^{3}=\left(\begin{array}{cc}
1 & 0 \\
0 & -1
\end{array}\right) .
$$

These are the Lie algebra generators of the fundamental representation of the four cover of $S O(4,2)$ :

$$
S U(2,2)=\left\{g=\left(\begin{array}{cc}
A & B \\
C & D
\end{array}\right) \in \operatorname{Mat}_{4 \times 4}(\mathbb{C}): g^{\dagger} \Gamma g=\Gamma, \operatorname{det}(g)=1\right\},
$$

with $\Gamma$ a $4 \times 4$ hermitian form of signature $(++--)$. In particular, taking $\Gamma=\gamma^{5}$, the $2 \times 2$ complex matrices $A, B, C, D$ in (2) satisfy the following restrictions:

$$
g^{-1} g=I_{4} \Leftrightarrow\left\{\begin{array}{c}
D^{\dagger} D-B^{\dagger} B=\sigma^{0} \\
A^{\dagger} A-C^{\dagger} C=\sigma^{0} \\
A^{\dagger} B-C^{\dagger} D=0
\end{array}\right.
$$

together with those of $g g^{-1}=I_{4}$. In this article we shall work with $S U(2,2)$ instead of $S O(4,2)$ and we shall use a set of complex coordinates to parametrize $S U(2,2)$. 
This parametrization will be adapted to the non-compact complex Grassmannian $\mathbb{D}_{4}=$ $U(2,2) / U(2)^{2}$ of the maximal compact subgroup $U(2)^{2}=U(2) \times U(2)$. It can be obtained through a block-orthonormalization process with metric $\Gamma=\gamma^{5}$ of the matrix columns of:

$$
\left(\begin{array}{cc}
\sigma^{0} & 0 \\
Z^{\dagger} & \sigma^{0}
\end{array}\right) \rightarrow g=\left(\begin{array}{cc}
\Delta_{1} & Z \Delta_{2} \\
Z^{\dagger} \Delta_{1} & \Delta_{2}
\end{array}\right),\left\{\begin{array}{l}
\Delta_{1}=\left(\sigma^{0}-Z Z^{\dagger}\right)^{-1 / 2}, \\
\Delta_{2}=\left(\sigma^{0}-Z^{\dagger} Z\right)^{-1 / 2}
\end{array}\right.
$$

Actually, we can identify

$$
Z=Z(g)=B D^{-1}, Z^{\dagger}=Z^{\dagger}(g)=C A^{-1}, \Delta_{1}=\left(A A^{\dagger}\right)^{1 / 2}, \Delta_{2}=\left(D D^{\dagger}\right)^{1 / 2} .
$$

From (3), we obtain the positive-matrix conditions $A A^{\dagger}>0$ and $D D^{\dagger}>0$, which are equivalent to:

$$
\sigma^{0}-Z Z^{\dagger}>0, \sigma^{0}-Z^{\dagger} Z>0
$$

and define the eight-dimensional symmetric complex Cartan-Bergman domain

$$
\mathbb{D}_{4}=U(2,2) / U(2)^{2}=\left\{Z \in \operatorname{Mat}_{2 \times 2}(\mathbb{C}): \sigma^{0}-Z Z^{\dagger}>0\right\}
$$

Moreover, the compactified Minkowski space $\mathbb{M}=\mathbb{S}^{3} \times_{\mathbb{Z}_{2}} \mathbb{S}^{1}$ is precisely the Shilov boundary $U(2)=\left\{Z \in \operatorname{Mat}_{2 \times 2}(\mathbb{C}): Z^{\dagger} Z=Z Z^{\dagger}=\sigma^{0}\right\}$ of $\mathbb{D}_{4}$.

There is a one-to-one mapping from $\mathbb{D}_{4}$ onto the future tube domain

$$
\mathbb{T}_{4}=\left\{W=X+i Y \in \operatorname{Mat}_{2 \times 2}(\mathbb{C}): Y>0\right\},
$$

of the complex Minkowski space $\mathbb{C}^{4}$, with $X=x_{\mu} \sigma^{\mu}$ and $Y=y_{\mu} \sigma^{\mu}$ hermitian matrices and $Y>0 \Leftrightarrow y_{0}>\|\vec{y}\|$. The (phase space) coordinates $x_{\mu}$ and $y_{\mu}$ are related to fourposition and four-momenta, respectively. This map is given by the Cayley transformation and its inverse:

$$
Z \rightarrow W(Z)=i\left(\sigma^{0}-Z\right)\left(\sigma^{0}+Z\right)^{-1}, \quad W \rightarrow Z(W)=\left(\sigma^{0}-i W\right)^{-1}\left(\sigma^{0}+i W\right) .
$$

This is the 3+1-dimensional analogue of the usual map from the unit disk onto the upper half-plane in two dimensions. Actually, the forward tube domain $\mathbb{T}_{4}$ is naturally homeomorphic to the quotient $U(2,2) / U(2)^{2}$ in a new realization of $U(2,2)$ in terms of complex $4 \times 4$ matrices $f$ which preserve $\Gamma=\gamma^{0}$ (that is, $f^{\dagger} \gamma^{0} f=\gamma^{0}$ ), instead of matrices $g$ preserving $\Gamma=\gamma^{5}$ and fulfilling (3). Both realizations of $U(2,2)$ are related by the map

$$
g \rightarrow f=\Upsilon g \Upsilon^{-1}, \Upsilon=\frac{1}{\sqrt{2}}\left(\begin{array}{cc}
\sigma^{0} & -\sigma^{0} \\
\sigma^{0} & \sigma^{0}
\end{array}\right) .
$$

In this article we shall mainly work with the $\Gamma=\gamma^{5}$ realization (the interested reader can see more details about the tube domain realization in, for example, 229] and [28]).

Let us proceed by giving a complete local parametrization of $U(2,2)$ adapted to the fibration $U(2)^{2} \rightarrow U(2,2) \rightarrow \mathbb{D}_{4}$. Any element $g \in G$ (in the present patch, containing the identity element) admits the Iwasawa decomposition

$$
g=\left(\begin{array}{cc}
A & B \\
C & D
\end{array}\right)=\left(\begin{array}{cc}
\Delta_{1} & Z \Delta_{2} \\
Z^{\dagger} \Delta_{1} & \Delta_{2}
\end{array}\right)\left(\begin{array}{cc}
U_{1} & 0 \\
0 & U_{2}
\end{array}\right),
$$


where the last factor $U_{1}=\Delta_{1}^{-1} A$ and $U_{2}=\Delta_{2}^{-1} D$ belongs to $U(2)^{2}$; i.e., $U_{1}, U_{2} \in U(2)$. Likewise, a parametrization of any $U \in U(2)$ (in a patch containing the identity), adapted to the quotient $\mathbb{S}^{2}=U(2) / U(1)^{2}$, is (the Hopf fibration)

$$
U=\left(\begin{array}{ll}
\mathrm{a} & \mathrm{b} \\
\mathrm{c} & \mathrm{d}
\end{array}\right)=\left(\begin{array}{cc}
\delta & z \delta \\
-\bar{z} \delta & \delta
\end{array}\right)\left(\begin{array}{cc}
u_{1} & 0 \\
0 & u_{2}
\end{array}\right),
$$

where $z=\mathrm{b} / \mathrm{d} \in \overline{\mathbb{C}} \simeq \mathbb{S}^{2}$ (the one-point compactification of $\mathbb{C}$ by inverse stereographic projection), $\delta=(1+z \bar{z})^{-1 / 2}$ and the phases $u_{1}=\mathrm{a} /|\mathrm{a}|, u_{2}=\mathrm{d} /|\mathrm{d}|$.

\section{Coherent states on $U(2,2) / U(2)^{2}$, closure relations and orthonormal basis}

Firstly, let us consider the Hilbert space $L^{2}(U(2,2), d \mu)$ of square integrable complex functions $\psi(g)$ on $U(2,2)$ with invariant scalar product

$$
\left\langle\psi \mid \psi^{\prime}\right\rangle=\int_{U(2,2)} d \mu(g) \psi(g) \overline{\psi^{\prime}(g)}
$$

given through the invariant Haar measure $d \mu(g)$, which can be decomposed as:

$$
\begin{aligned}
d \mu(g) & =\left.\left.d \mu(g)\right|_{\mathbb{D}_{4}} d \mu(g)\right|_{U(2)^{2}}, \\
\left.d \mu(g)\right|_{\mathbb{D}_{4}} & =\operatorname{det}\left(\sigma_{0}-Z^{\dagger} Z\right)^{-4}|d Z|, \\
\left.d \mu(g)\right|_{U(2)^{2}} & =d v\left(U_{1}\right) d v\left(U_{2}\right),
\end{aligned}
$$

where we are denoting by $d v(U)$ the Haar measure on $U(2)$, which can be in turn decomposed as:

$$
\begin{aligned}
d v(U) & =\left.\left.d v(U)\right|_{\mathbb{S}^{2}} d v(U)\right|_{U(1)^{2}}, \\
\left.d v(U)\right|_{\mathbb{S}^{2}} & =(1+z \bar{z})^{-2}|d z|, \\
\left.d v(U)\right|_{U(1)^{2}} & =-\bar{u}_{1} d u_{1} \bar{u}_{2} d u_{2} .
\end{aligned}
$$

We have used the Iwasawa decomposition of an element $g$ given in (11]12) and denoted by $|d z|$ and $|d Z|$ the Lebesgue measures on $\mathbb{C}$ and $\mathbb{C}^{4}$, respectively (see [28] for more explicit expressions of this measure). The group $U(2,2)$ is represented on $L^{2}(U(2,2), d \mu)$ as (left-action) $\left[\mathcal{U}\left(g^{\prime}\right) \psi\right](g)=\psi\left(g^{\prime-1} g\right)$. This representation is reducible and we shall restrict it to an irreducible subspace. As we want to restrict ourselves to the quotient $U(2,2) / U(2)^{2}$, we chose as fiducial (ground state, lowest weight) vector $\psi_{0}^{\lambda}(g) \equiv \operatorname{det}(D)^{-\lambda}$ for $g$ given in (11) and $\lambda$ an integer number that will eventually label the corresponding irreducible representation (the so called "scale dimension" [26], which is also related to the helicity as we comment later in Sec. 5). In fact, $\psi_{0}^{\lambda}(g)$ is invariant (up to a phase) under $U(2)^{2} \subset U(2,2)$ since, for $g^{\prime}=\left(\begin{array}{cc}U_{1} & 0 \\ 0 & U_{2}\end{array}\right) \in U(2)^{2}$, we have

$$
\psi_{0}^{\lambda}\left(g^{\prime-1} g\right)=\operatorname{det}\left(U_{2}^{\dagger} D\right)^{-\lambda}=\operatorname{det}\left(U_{2}^{\dagger}\right)^{-\lambda} \psi_{0}^{\lambda}(g) .
$$


Under a general element $g^{\prime}=\left(\begin{array}{cc}A^{\prime} & B^{\prime} \\ C^{\prime} & D^{\prime}\end{array}\right) \in U(4)$, the vector $\psi_{0}^{\lambda}$ transforms as

$$
\psi_{g^{\prime}}^{\lambda}(g) \equiv \psi_{0}^{\lambda}\left(g^{\prime-1} g\right)=\operatorname{det}\left(D^{\prime \dagger} D-B^{\prime \dagger} B\right)^{\lambda}=\operatorname{det}\left(D^{\prime \dagger}-B^{\prime \dagger} Z\right)^{\lambda} \psi_{0}^{\lambda}(g),
$$

where we have used the relations (5) to write $Z=B D^{-1}$. The set of functions in the orbit of $\psi_{0}^{\lambda}$ under $U(2,2)$

$$
\mathcal{S}_{\lambda}=\left\{\psi_{g}^{\lambda} \equiv \mathcal{U}(g) \psi_{0}^{\lambda}, g \in U(2,2)\right\}
$$

defines a system of CS. Note that $\psi_{g}^{\lambda}$ and $\psi_{g^{\prime}}^{\lambda}$ are equivalent (up to a phase) if $g^{\prime} g^{\dagger} \in$ $U(2)^{2} \subset U(2,2)$. We shall prove that this coherent state system fulfills the resolution of the identity

$$
1=\left.c_{\lambda} \int_{\mathbb{D}_{4}} d \mu(g)\right|_{\mathbb{D}_{4}}\left|\psi_{g}^{\lambda}\right\rangle\left\langle\psi_{g}^{\lambda}\right|,
$$

with a suitable normalization constant $c_{\lambda}$. Before, let us briefly review some auxiliary results. Note that, introducing $Z^{\prime \dagger}=D^{\prime \dagger-1} B^{\prime \dagger}$ as in (5), the state (17) can be written as

$$
\psi_{g^{\prime}}^{\lambda}(g)=\operatorname{det}\left(\sigma_{0}-Z^{\prime \dagger} Z\right)^{\lambda} \overline{\psi_{0}^{\lambda}\left(g^{\prime}\right)} \psi_{0}^{\lambda}(g)
$$

We also realize that $\left|\psi_{0}^{\lambda}(g)\right|^{2}=\operatorname{det}\left(D D^{\dagger}\right)^{-\lambda}=\operatorname{det}\left(\sigma_{0}-Z^{\dagger} Z\right)^{-\lambda}$. To prove (19), we would like to have before an expansion of $\operatorname{det}\left(\sigma_{0}-Z^{\prime \dagger} Z\right)^{\lambda}$ in terms of orthogonal polynomials. The following identity was proved in [28].

Lemma 3.1. Let us denote by

$$
\begin{aligned}
& \mathcal{D}_{q_{a}, q_{b}}^{j}(X)=\sqrt{\frac{\left(j+q_{a}\right) !\left(j-q_{a}\right) !}{\left(j+q_{b}\right) !\left(j-q_{b}\right) !}} \sum_{k=\max \left(0, q_{a}+q_{b}\right)}^{\min \left(j+q_{a}, j+q_{b}\right)}\left(\begin{array}{c}
j+q_{b} \\
k
\end{array}\right)\left(\begin{array}{c}
j-q_{b} \\
k-q_{a}-q_{b}
\end{array}\right) \\
& \times x_{11}^{k} x_{12}^{j+q_{a}-k} x_{21}^{j+q_{b}-k} x_{22}^{k-q_{a}-q_{b}},
\end{aligned}
$$

the usual Wigner's $\mathcal{D}$-matrices for $S U(2)$ (see e.g. [8]), where $j \in \mathbb{N} / 2$ (the spin) runs on all non-negative half-integers and $q_{a}, q_{b}=-j,-j+1, \ldots, j-1, j$, and $X$ represents here an arbitrary $2 \times 2$ complex matrix with entries $x_{u v}$. For every $\lambda \in \mathbb{N}, \lambda \geq 2$, the following identity holds:

$$
\begin{array}{r}
\sum_{j \in \mathbb{N} / 2} \frac{2 j+1}{\lambda-1} \sum_{m=0}^{\infty} t^{2 j+2 m}\left(\begin{array}{c}
m+\lambda-2 \\
\lambda-2
\end{array}\right)\left(\begin{array}{c}
m+2 j+\lambda-1 \\
\lambda-2
\end{array}\right) \operatorname{det}(X)^{m} \sum_{q=-j}^{j} \mathcal{D}_{q q}^{j}(X) \\
=\operatorname{det}\left(\sigma^{0}-t X\right)^{-\lambda}
\end{array}
$$

where the sum on $j$ runs over all half-nonnegative integers: $j=0, \frac{1}{2}, 1, \frac{3}{2}, 2, \ldots$. From this Lemma, the following interesting result can be easily proved. 
Theorem 3.2. The infinite set of homogeneous polynomials

$$
\varphi_{q_{1}, q_{2}}^{j, m}(Z)=\sqrt{\frac{2 j+1}{\lambda-1}\left(\begin{array}{c}
m+\lambda-2 \\
\lambda-2
\end{array}\right)\left(\begin{array}{c}
m+2 j+\lambda-1 \\
\lambda-2
\end{array}\right)} \operatorname{det}(Z)^{m} \mathcal{D}_{q_{1}, q_{2}}^{j}(Z),
$$

of degree $2 j+2 m$ verifies the following closure relation (the reproducing Bergman kernel):

$$
\sum_{j \in \mathbb{N} / 2} \sum_{m=0}^{\infty} \sum_{q_{1}, q_{2}=-j}^{j} \overline{\varphi_{q_{1}, q_{2}}^{j, m}(Z)} \varphi_{q_{1}, q_{2}}^{j, m}\left(Z^{\prime}\right)=\frac{1}{\operatorname{det}\left(\sigma^{0}-Z^{\dagger} Z^{\prime}\right)^{\lambda}}
$$

and constitutes an orthonormal basis of Hilbert space $\mathcal{H}_{\lambda}\left(\mathbb{D}_{4}\right)=L_{h}^{2}\left(\mathbb{D}_{4}, d \mu_{\lambda}\right)$ of analytic square-integrable holomorphic functions on $\mathbb{D}_{4}$ with measure

$$
\left.d \mu_{\lambda}\left(Z, Z^{\dagger}\right) \equiv c_{\lambda}\left|\psi_{0}^{\lambda}(g)\right|^{2} d \mu(g)\right|_{\mathbb{D}_{4}}=c_{\lambda} \operatorname{det}\left(\sigma_{0}-Z^{\dagger} Z\right)^{\lambda-4}|d Z|
$$

where $c_{\lambda}=(\lambda-1)(\lambda-2)^{2}(\lambda-3) / \pi^{4}$ is a normalization constant and $\lambda>3$.

This theorem has been proved in [28] in the context of conformal wavelets. Here we only point out that, replacing $X=Z^{\prime \dagger} Z$ in (22) and using determinant and Wigner's $\mathcal{D}$-matrix properties [8], one easily realizes that that (22) reproduces (24).

Let us introduce bracket notation and put

$$
\left\langle{ }_{q_{a}, q_{b}}^{j, m} \mid Z\right\rangle \equiv \varphi_{q_{a}, q_{b}}^{j, m}(Z) \operatorname{det}\left(\sigma_{0}-Z^{\dagger} Z\right)^{\lambda / 2} .
$$

(We remove the label $\lambda$ from the definition of $\left|\underset{q_{a}, q_{b}}{j, m}\right\rangle$ for the sake of brevity). This makes $\mathcal{H}_{\lambda}\left(\mathbb{D}_{4}\right)$ a reproducing kernel Hilbert space, that is, a Hilbert space of functions $\varphi$ in which pointwise evaluation $\varphi(Z)$ is a continuous linear functional. The resolution of the identity for an orthonormal basis in $\mathcal{H}_{\lambda}\left(\mathbb{D}_{4}\right)$ then adopts the form

$$
\left.1=\sum_{m=0}^{\infty} \sum_{j \in \mathbb{N} / 2} \sum_{q_{a}, q_{b}=-j}^{j}||_{q_{a}, q_{b}}^{j, m}\right\rangle\left\langle j_{q_{a}, q_{b}}^{j, m}\right|
$$

and the formal ket $|Z\rangle$ is

$$
\left.|Z\rangle=\left.\operatorname{det}\left(\sigma_{0}-Z^{\dagger} Z\right)^{\lambda / 2} \sum_{m=0}^{\infty} \sum_{j \in \mathbb{N} / 2} \sum_{q_{a}, q_{b}=-j}^{j} \varphi_{q_{a}, q_{b}}^{j, m}(Z)\right|_{q_{a}, q_{b}} ^{j, m}\right\rangle .
$$

Actually, we can identify $|Z\rangle$ with the coherent state $\left|\psi_{g}^{\lambda}\right\rangle$ in (17) up to a phase. From the coherent state overlap

$$
\left\langle Z^{\prime} \mid Z\right\rangle=\frac{\operatorname{det}\left(\sigma_{0}-Z^{\prime \dagger} Z^{\prime}\right)^{\lambda / 2} \operatorname{det}\left(\sigma_{0}-Z^{\dagger} Z\right)^{\lambda / 2}}{\operatorname{det}\left(\sigma_{0}-Z^{\prime \dagger} Z\right)^{\lambda}}
$$

we see that $|Z\rangle$ is normalized. Moreover, using the orthogonality properties of the homogeneous polynomials $\varphi_{q_{a}, q_{b}}^{j, m}(Z)$, it is direct to prove the announced resolution of unity (19), now written as:

$$
1=c_{\lambda} \int_{\mathbb{D}_{4}}|Z\rangle\left\langle Z|d \mu(g)|_{\mathbb{D}_{4}} .\right.
$$


It is interesting to compare the $U(2,2) / U(2)^{2} \mathrm{CS}(28)$ with the well known $U(1,1) / U(1)^{2}$, or Bargmann index- $\kappa$ (with $\kappa>1 / 2)$, CS

$$
|z\rangle=\left(1-|z|^{2}\right)^{\kappa} \sum_{n=0}^{\infty} \varphi_{n}(z)|\kappa, n\rangle, \quad \varphi_{n}(z)=\left(\begin{array}{c}
2 \kappa+n-1 \\
n
\end{array}\right)^{1 / 2} z^{n},
$$

with $z \in \mathbb{D}_{1}=\left\{z \in \mathbb{C}: 1-|z|^{2}>0\right\}$ (the stereographic projection of the hyperboloid $U(1,1) / U(1)^{2}$ onto the unit disk), for which the coherent state overlap and the resolution of the identity acquire the form

$$
\left\langle z^{\prime} \mid z\right\rangle=\frac{\left(1-\left|z^{\prime}\right|^{2}\right)^{\kappa}\left(1-|z|^{2}\right)^{\kappa}}{\left(1-\bar{z}^{\prime} z\right)^{2 \kappa}}, \quad 1=\frac{2 \kappa-1}{\pi} \int_{\mathbb{D}_{1}}|z\rangle\langle z| \frac{|d z|}{\left(1-|z|^{2}\right)^{2}} .
$$

We perceive a similar structure between $U(2,2) / U(2)^{2}$ and $U(1,1) / U(1)^{2} \mathrm{CS}$, although the case $U(2,2) / U(2)^{2}$ is more involved and can be regarded as a generalized (matrix $Z$ ) version of the standard (scalar $z$ ) case.

We finish this section with an explicit form of the unirep of $U(2,2)$ on $\mathcal{H}_{\lambda}\left(\mathbb{D}_{4}\right)$ in the form of a Corollary.

Corollary 3.3. For any holomorphic function $\phi \in \mathcal{H}_{\lambda}\left(\mathbb{D}_{4}\right)$ and any $g^{\prime} \in U(2,2)$, the following action

$$
\left[\mathcal{U}_{g^{\prime}}^{\lambda} \phi\right](Z) \equiv \operatorname{det}\left(D^{\prime \dagger}-B^{\prime \dagger} Z\right)^{-\lambda} \phi\left(Z^{\prime}\right), Z^{\prime}=\left(A^{\prime \dagger} Z-C^{\prime \dagger}\right)\left(D^{\prime \dagger}-B^{\prime \dagger} Z\right)^{-1}
$$

defines a square-integrable unitary irreducible representation of $U(2,2)$ on $\mathcal{H}_{\lambda}\left(\mathbb{D}_{4}\right)$. Note that if we define $\psi(g) \equiv \psi_{0}^{\lambda}(g) \phi(Z), Z=Z(g)$, then

$$
\left[\mathcal{U}_{g^{\prime}}^{\lambda} \phi\right](Z)=\left(\psi_{0}^{\lambda}(g)\right)^{-1}\left[\mathcal{U}\left(g^{\prime}\right) \psi\right](g) .
$$

The unitarity of $\mathcal{U}$ in $L^{2}(U(2,2), d \mu)$ directly implies the unitarity of $\mathcal{U}^{\lambda}$ in $\mathcal{H}\left(\mathbb{D}_{4}\right)$. Irreducibility follows from the fact that, for example, for $\phi(Z)=1$, the transformed function

$$
\left[\mathcal{U}_{g^{\prime}}^{\lambda} \phi\right](Z) \equiv \operatorname{det}\left(D^{\prime \dagger}-B^{\prime \dagger} Z\right)^{\lambda}=\sum_{m=0}^{\infty} \sum_{j \in \mathbb{N} / 2} \sum_{q_{a}, q_{b}=-j}^{j} c_{q_{a}, q_{b}}^{j, m}\left(g^{\prime}\right) \varphi_{q_{a}, q_{b}}^{j, m}(Z)
$$

is expanded in terms of all basis functions $\varphi_{q_{a}, q_{b}}^{j, m}(Z)$ with non-zero coefficients $c_{q_{a}, q_{b}}^{j, m}\left(g^{\prime}\right)=$ $\operatorname{det}\left(D^{\prime \dagger}\right)^{-\lambda} \overline{\varphi_{q_{a}, q_{b}}^{j, m}\left(B^{\prime} D^{\prime-1}\right)}$, as follows from (24).

Remark 3.4. Instead of the ground state $\psi_{0}^{\lambda}(g)=\operatorname{det}(D)^{-\lambda}$, we could also have chosen $\psi_{0}^{\lambda}(g)=\operatorname{det}(A)^{-\lambda}$, for which we would have arrived to a square-integrable unitary irrep of $U(2,2)$ on the space $\overline{\mathcal{H}_{\lambda}\left(\mathbb{D}_{4}\right)}$ of anti-holomorphic functions $\phi\left(Z^{\dagger}\right)$. 


\section{Infinitesimal generators, matrix elements and op- erator symbols}

Let us denote by $\mathfrak{P}^{\mu}, \mathfrak{M}_{\mu \nu}, \mathfrak{D}$ and $\mathfrak{K}^{\mu}$ the infinitesimal (differential) generators of the finite action (33) fulfilling the same commutation relations as the matrix generators $P^{\mu}, M^{\mu \nu}, D$ and $K^{\mu}$ in (11). Writting $Z=z_{\mu} \sigma^{\mu}, z_{\mu} \in \mathbb{C}, z^{2}=z_{\mu} z^{\mu}, \partial^{\mu}=\partial / \partial z_{\mu}$, these generators have the following expression:

$$
\begin{array}{r}
\mathfrak{M}^{\mu \nu}=z^{\mu} \partial^{\nu}-z^{\nu} \partial^{\mu}, \quad \mathfrak{D}=z_{\mu} \partial^{\mu}+\lambda, \\
\mathfrak{P}^{\mu}=\partial^{\mu}, \quad \mathfrak{K}^{\mu}=z^{2} \mathfrak{P}^{\mu}-2 z^{\mu} \mathfrak{D},
\end{array}
$$

Let us compute their action on the orthonormal basis functions (23). Firstly we see that the homogeneous polynomials in (23) are eigenfunctions of the dilation generator $\mathfrak{D}$

$$
\mathfrak{D} \varphi_{q_{a}, q_{b}}^{j, m}=(2 j+2 m+\lambda) \varphi_{q_{a}, q_{b}}^{j, m},
$$

with eigenvalue $2 j+2 m+\lambda$, where $2 j+2 m$ is the homogeneity degree of $\varphi_{q_{a}, q_{b}}^{j, m}$ and $\lambda$ is the scale dimension. Similarly, we can compute the infinitesimal action of spacetime translations

$$
\begin{aligned}
\mathfrak{P}^{0} \varphi_{q_{a}, q_{b}}^{j, m}= & C_{q_{a}, q_{b}}^{j, m+2 j+1} \varphi_{q_{a}-\frac{1}{2}, q_{b}-\frac{1}{2}}^{j-\frac{1}{2}, m}+C_{-q_{a}+\frac{1}{2},-q_{b}+\frac{1}{2}}^{j+\frac{1}{2}, m} \varphi_{q_{a}-\frac{1}{2}, q_{b}-\frac{1}{2}}^{j+\frac{1}{2}, m-1}+ \\
& C_{-q_{a},-q_{b}}^{j, m+2 j+1} \varphi_{q_{a}+\frac{1}{2}, q_{b}+\frac{1}{2}}^{j-\frac{1}{2}, m}+C_{q_{a}+\frac{1}{2}, q_{b}+\frac{1}{2}}^{j+\frac{1}{2}, m} \varphi_{q_{a}+\frac{1}{2}, q_{b}+\frac{1}{2}}^{j+\frac{1}{2}, m-1}, \\
\mathfrak{P}^{1} \varphi_{q_{a}, q_{b}}^{j, m}= & C_{-q_{a}, q_{b}}^{j, m+2 j+1} \varphi_{q_{a}+\frac{1}{2}, q_{b}-\frac{1}{2}}^{j-\frac{1}{2}, m}-C_{q_{a}+\frac{1}{2},-q_{b}+\frac{1}{2}}^{j+\frac{1}{2}, m} \varphi_{q_{a}+\frac{1}{2}, q_{b}-\frac{1}{2}}^{j+\frac{1}{2}, m-1}+ \\
& C_{q_{a},-q_{b}}^{j, m+2 j+1} \varphi_{q_{a}-\frac{1}{2}, q_{b}+\frac{1}{2}}^{j-\frac{1}{2}, m}-C_{-q_{a}+\frac{1}{2}, q_{b}+\frac{1}{2}}^{j+\frac{1}{2}, m} \varphi_{q_{a}-\frac{1}{2}, q_{b}+\frac{1}{2}}^{j+\frac{1}{2}, m-1}, \\
\mathfrak{P}^{2} \varphi_{q_{a}, q_{b}}^{j, m}= & i C_{-q_{a}, q_{b}}^{j, m+2 j+1} \varphi_{q_{a}+\frac{1}{2}, q_{b}-\frac{1}{2}}^{j-\frac{1}{2}, m}-i C_{q_{a}+\frac{1}{2},-q_{b}+\frac{1}{2}}^{j+\frac{1}{2}, m} \varphi_{q_{a}+\frac{1}{2}, q_{b}-\frac{1}{2}}^{j+\frac{1}{2}, m-1}- \\
& i C_{q_{a},-q_{b}}^{j, m+2 j+1} \varphi_{q_{a}-\frac{1}{2}, q_{b}+\frac{1}{2}}^{j-\frac{1}{2}, m}+i C_{-q_{a}+\frac{1}{2}, q_{b}+\frac{1}{2}}^{j+\frac{1}{2}, m} \varphi_{q_{a}-\frac{1}{2}, q_{b}+\frac{1}{2}}^{j+\frac{1}{2}, m-1}, \\
\mathfrak{P}^{3} \varphi_{q_{a}, q_{b}}^{j, m}= & C_{q_{a}, q_{b}}^{j, m+2 j+1} \varphi_{q_{a}-\frac{1}{2}, q_{b}-\frac{1}{2}}^{j-\frac{1}{2}, m}+C_{-q_{a}+\frac{1}{2},-q_{b}+\frac{1}{2}}^{j+\frac{1}{2}, m} \varphi_{q_{a}-\frac{1}{2}, q_{b}-\frac{1}{2}}^{j+\frac{1}{2}, m-1}- \\
& C_{-q_{a},-q_{b}}^{j, m+2 j+1} \varphi_{q_{a}+\frac{1}{2}, q_{b}+\frac{1}{2}}^{j-\frac{1}{2}, m}-C_{q_{a}+\frac{1}{2}, q_{b}+\frac{1}{2}}^{j+\frac{1}{2}, m} \varphi_{q_{a}+\frac{1}{2}, q_{b}+\frac{1}{2}}^{j+\frac{1}{2}, m-1},
\end{aligned}
$$


and relativistic uniform accelerations

$$
\begin{aligned}
\mathfrak{K}^{0} \varphi_{q_{a}, q_{b}}^{j, m}= & -C_{q_{a}, q_{b}}^{j, m+1} \varphi_{q_{a}-\frac{1}{2}, q_{b}-\frac{1}{2}}^{j-\frac{1}{2}, m+1}-C_{-q_{a},-q_{b}}^{j, m+1} \varphi_{q_{a}+\frac{1}{2}, q_{b}+\frac{1}{2}}^{j-\frac{1}{2}, m+1}- \\
& C_{-q_{a}+\frac{1}{2},-q_{b}+\frac{1}{2}}^{j+\frac{1}{2}, m+2 j+1} \varphi_{q_{a}-\frac{1}{2}, q_{b}-\frac{1}{2}}^{j+\frac{1}{2}, m}-C_{q_{a}+\frac{1}{2}, q_{b}+\frac{1}{2}}^{j+\frac{1}{2}, m+2 j+1} \varphi_{q_{a}+\frac{1}{2}, q_{b}+\frac{1}{2}}^{j+\frac{1}{2}, m}, \\
\mathfrak{K}^{1} \varphi_{q_{a}, q_{b}}^{j, m}= & C_{-q_{a}+\frac{1}{2}, q_{b}+\frac{1}{2}}^{j+\frac{1}{2}, m+2 j+1} \varphi_{q_{a}-\frac{1}{2}, q_{b}+\frac{1}{2}}^{j+\frac{1}{2}, m}+C_{q_{a}+\frac{1}{2},-q_{b}+\frac{1}{2}}^{j+\frac{1}{2}, m+2 j+1} \varphi_{q_{a}+\frac{1}{2}, q_{b}-\frac{1}{2}}^{j+\frac{1}{2}, m}- \\
& C_{q_{a},-q_{b}}^{j, m+1} \varphi_{q_{a}-\frac{1}{2}, q_{b}+\frac{1}{2}}^{j-\frac{1}{2}, m+1}-C_{-q_{a}, q_{b}}^{j, m+1} \varphi_{q_{a}+\frac{1}{2}, q_{b}-\frac{1}{2}}^{j-\frac{1}{2}, m+1}, \\
\mathfrak{K}^{2} \varphi_{q_{a}, q_{b}}^{j, m}= & -i C_{-q_{a}+\frac{1}{2}, q_{b}+\frac{1}{2}}^{j+\frac{1}{2}, m+2 j+1} \varphi_{q_{a}-\frac{1}{2}, q_{b}+\frac{1}{2}}^{j+\frac{1}{2}, m}+i C_{q_{a}+\frac{1}{2},-q_{b}+\frac{1}{2}}^{j+\frac{1}{2}, m+2 j+1} \varphi_{q_{a}+\frac{1}{2}, q_{b}-\frac{1}{2}}^{j+\frac{1}{2}, m} \\
& i C_{q_{a},-q_{b}}^{j, m+1} \varphi_{q_{a}-\frac{1}{2}, q_{b}+\frac{1}{2}}^{j-\frac{1}{2}, m+1}-i C_{-q_{a}, q_{b}}^{j, m+1} \varphi_{q_{a}+\frac{1}{2}, q_{b}-\frac{1}{2}}^{j-\frac{1}{2}, m+1}, \\
\mathfrak{K}^{3} \varphi_{q_{a}, q_{b}}^{j, m}= & C_{q_{a}+\frac{1}{2}, q_{b}+\frac{1}{2}}^{j+\frac{1}{2}, m+2 j+1} \varphi_{q_{a}+\frac{1}{2}, q_{b}+\frac{1}{2}}^{j+\frac{1}{2}, m}-C_{-q_{a}+\frac{1}{2},-q_{b}+\frac{1}{2}}^{j+\frac{1}{2}, m+2 j+1} \varphi_{q_{a}-\frac{1}{2}, q_{b}-\frac{1}{2}}^{j+\frac{1}{2}, m}+ \\
& C_{-q_{a},-q_{b}}^{j, m+1} \varphi_{q_{a}+\frac{1}{2}, q_{b}+\frac{1}{2}}^{j-\frac{1}{2}, m+1}-C_{q_{a}, q_{b}}^{j, m+1} \varphi_{q_{a}-\frac{1}{2}, q_{b}-\frac{1}{2}}^{j-\frac{1}{2}, m+1},
\end{aligned}
$$

with

$$
C_{q_{a}, q_{b}}^{j, m} \equiv \frac{\sqrt{\left(j+q_{a}\right)\left(j+q_{b}\right) m(\lambda+m-2)}}{\sqrt{2 j(2 j+1)}} .
$$

The infinitesimal generators of rotations $U(2)^{2}=U_{a}(2) \times U_{b}(2)$ are the angular momentum operators $\mathfrak{S}_{a j}=\frac{1}{2}\left(\mathfrak{M}_{0 j}-i \epsilon_{j k l} \mathfrak{M}_{k l}\right)$ and $\mathfrak{S}_{b j}=\frac{1}{2}\left(\mathfrak{M}_{0 j}+i \epsilon_{j k l} \mathfrak{M}_{k l}\right)$. The action of the angular-momentum third component is

$$
\mathfrak{S}_{\ell 3} \varphi_{q_{a}, q_{b}}^{j, m}=q_{\ell} \varphi_{q_{a}, q_{b}}^{j, m}, \ell=a, b
$$

and the action of the ladder angular-momentum operators is

$$
\mathfrak{S}_{\ell \pm} \varphi_{q_{a}, q_{b}}^{j, m}=\sqrt{\left(j \mp q_{\ell}\right)\left(j \pm q_{\ell}+1\right)} \varphi_{q_{a} \pm \delta_{\ell, a}, q_{b} \pm \delta_{\ell, b}}^{j, m}, \quad \ell=a, b
$$

where $\mathfrak{S}_{a \pm}=\mathfrak{S}_{a 1} \mp i \mathfrak{S}_{a 2}$ and $\mathfrak{S}_{b \pm}=\mathfrak{S}_{b 1} \pm i \mathfrak{S}_{b 2}$. Note that $\mathfrak{S}_{a \pm}$ and $\mathfrak{S}_{b \pm}$ have conjugated definitions $( \pm \leftrightarrow \mp)$. This fact is related to the transformation property of wave functions in (33) which, for pure rotations $\left(C^{\prime}=0=B^{\prime}, A^{\prime}=V_{a}, D^{\prime}=V_{b} ; V_{\ell} \in S U(2), \ell=a, b\right)$ gives $\left[\mathcal{U}_{g^{\prime}}^{\lambda} \phi\right](Z)=\phi\left(V_{a}^{\dagger} Z V_{b}\right)$, so that rotations $V_{a}$ are represented by the inverse $V_{a}^{\dagger}$.

For completeness, we also give the action of $U(2)^{2}$-invariant (i.e., commuting with $\mathfrak{M}_{\mu \nu}$ ) quadratic operators:

$$
\begin{aligned}
\mathfrak{M}_{\mu \nu} \mathfrak{M}^{\mu \nu} \varphi_{q_{a}, q_{b}}^{j, m} & =-8 j(j+1) \varphi_{q_{a}, q_{b}}^{j, m}, \\
\mathfrak{P}^{\mu} \mathfrak{P}_{\mu} \varphi_{q_{a}, q_{b}}^{j, m} & =4 \sqrt{m(2 j+m+1)(\lambda+m-2)(\lambda+2 j+m-1)} \varphi_{q_{a}, q_{b}}^{j, m-1}, \\
\mathfrak{K}^{\mu} \mathfrak{K}_{\mu} \varphi_{q_{a}, q_{b}}^{j, m} & =4 \sqrt{(m+1)(2 j+m+2)(\lambda+m-1)(\lambda+2 j+m)} \varphi_{q_{a}, q_{b}}^{j, m+1}, \\
\mathfrak{K}^{\mu} \mathfrak{P}_{\mu} \varphi_{q_{a}, q_{b}}^{j, m} & =-4\left(2 j^{2}+m(m+\lambda-2)+j(2 m+\lambda-1)\right) \varphi_{q_{a}, q_{b}}^{j, m}, \\
\mathfrak{P}^{\mu} \mathfrak{K}_{\mu} \varphi_{q_{a}, q_{b}}^{j, m} & =-4\left(2 j^{2}+(m+2)(m+\lambda)+j(2 m+\lambda+3)\right) \varphi_{q_{a}, q_{b}}^{j, m} .
\end{aligned}
$$


One can verify that $\mathfrak{P}^{\mu} \mathfrak{K}_{\mu}+\mathfrak{K}^{\mu} \mathfrak{P}_{\mu}=2 \mathfrak{P}^{\mu} \mathfrak{K}_{\mu}+8 \mathfrak{D}$, as deduced from the original commutation relation $\left[K_{\mu}, P_{\nu}\right]=2\left(\eta_{\mu \nu} D+M_{\mu \nu}\right)$. With these ingredients, the value of the quadratic Casimir operator

$$
\mathfrak{C}_{2}=\mathfrak{D}^{2}-\frac{1}{2} \mathfrak{M}_{\mu \nu} \mathfrak{M}^{\mu \nu}+\frac{1}{2}\left(\mathfrak{P}_{\mu} \mathfrak{K}^{\mu}+\mathfrak{K}_{\mu} \mathfrak{P}^{\mu}\right)
$$

in the Hilbert space $\mathcal{H}_{\lambda}\left(\mathbb{D}_{4}\right)$ is easily computed and gives:

$$
\mathfrak{C}_{2} \varphi_{q_{a}, q_{b}}^{j, m}=\lambda(\lambda-4) \varphi_{q_{a}, q_{b}}^{j, m}, \quad \forall j, m, q_{a}, q_{b}
$$

We shall also provide the operator symbols (the expectation value in $|Z\rangle$ ) of the previous operators. Given the differential representation (36) of any operator $\mathfrak{O}$ we can simply compute its symbol as

$$
\langle\mathfrak{O}\rangle \equiv\langle Z|\mathfrak{O}| Z\rangle=\frac{\mathfrak{O} \operatorname{det}\left(\sigma^{0}-Z^{\dagger} Z\right)^{-\lambda}}{\operatorname{det}\left(\sigma^{0}-Z^{\dagger} Z\right)^{-\lambda}} .
$$

With this information, the operator symbols of (36) and their quadratic scalar combinations (43) are

$$
\begin{gathered}
\langle\mathfrak{D}\rangle=\lambda \frac{1-\operatorname{det}\left(Z^{\dagger} Z\right)}{\operatorname{det}\left(\sigma^{0}-Z^{\dagger} Z\right)},\left\langle\mathfrak{P}^{\mu}\right\rangle=2 \lambda \frac{\bar{z}_{\mu}-\operatorname{det}\left(Z^{\dagger} Z\right) z^{\mu}}{\operatorname{det}\left(\sigma^{0}-Z^{\dagger} Z\right)}, \\
\left\langle\mathfrak{K}^{\mu}\right\rangle=\operatorname{det}\left(Z^{\dagger} Z\right)\left\langle\mathfrak{P}^{\mu}\right\rangle-2 z^{\mu}\langle\mathfrak{D}\rangle,\left\langle\mathfrak{M}^{\mu \nu}\right\rangle=z^{\mu}\left\langle\mathfrak{P}^{\nu}\right\rangle-z^{\nu}\left\langle\mathfrak{P}^{\mu}\right\rangle, \\
\left\langle\mathfrak{D}^{2}\right\rangle=\frac{\lambda+1}{\lambda}\langle\mathfrak{D}\rangle^{2}-\lambda \frac{1+\operatorname{det}\left(Z^{\dagger} Z\right)}{\operatorname{det}\left(\sigma^{0}-Z^{\dagger} Z\right)},\left\langle\mathfrak{P}^{\mu} \mathfrak{P}_{\mu}\right\rangle=\frac{4 \lambda(\lambda-1) \operatorname{det}\left(Z^{\dagger}\right)}{\operatorname{det}\left(\sigma^{0}-Z^{\dagger} Z\right)},\left\langle\mathfrak{K}^{\mu} \mathfrak{K}_{\mu}\right\rangle=\frac{4 \lambda(\lambda-1) \operatorname{det}(Z)}{\operatorname{det}\left(\sigma^{0}-Z^{\dagger} Z\right)}, \\
\left\langle\mathfrak{P}^{\mu} \mathfrak{K}_{\mu}\right\rangle=2\left(\lambda \frac{\lambda-3+\operatorname{tr}\left(Z^{\dagger} Z\right)+(1+\lambda) \operatorname{det}\left(Z^{\dagger} Z\right)}{\operatorname{det}\left(\sigma^{0}-Z^{\dagger} Z\right)}-\frac{\lambda+1}{\lambda}\langle\mathfrak{D}\rangle^{2}\right), \\
\left\langle\mathfrak{K}^{\mu} \mathfrak{P}_{\mu}\right\rangle=\left\langle\mathfrak{P}^{\mu} \mathfrak{K}_{\mu}\right\rangle+8\langle\mathfrak{D}\rangle,\left\langle\mathfrak{M}_{\mu \nu} \mathfrak{M}^{\mu \nu}\right\rangle=2\left\langle\mathfrak{D}^{2}\right\rangle+\left\langle\mathfrak{K}^{\mu} \mathfrak{P}_{\mu}\right\rangle+\left\langle\mathfrak{P}^{\mu} \mathfrak{K}_{\mu}\right\rangle-2 \lambda(\lambda-4),
\end{gathered}
$$

where the last one is a consequence of (44) and (45). Actually, one can verify that the star commutator of symbols $\left[\left\langle\mathfrak{O}_{1}\right\rangle,\left\langle\mathfrak{O}_{2}\right\rangle\right]_{*} \equiv\left\langle\mathfrak{O}_{1}\right\rangle *\left\langle\mathfrak{O}_{2}\right\rangle-\left\langle\mathfrak{O}_{2}\right\rangle *\left\langle\mathfrak{O}_{1}\right\rangle$, with star product $\left\langle\mathfrak{O}_{1}\right\rangle *\left\langle\mathfrak{O}_{2}\right\rangle \equiv\left\langle\mathfrak{O}_{1} \mathfrak{O}_{2}\right\rangle$, defines a representation $\left[\left\langle\mathfrak{O}_{1}\right\rangle,\left\langle\mathfrak{O}_{2}\right\rangle\right]_{*}=\left\langle\left[\mathfrak{O}_{1}, \mathfrak{O}_{2}\right]\right\rangle$ of the $\operatorname{su}(2,2)$ Lie algebra.

\section{Oscillator realization, massive compounds and ex- citons}

It is well known the oscillator (Jordan-Schwinger) realization of the $S U(1,1)$ generators $\mathcal{Q}_{3}, \mathcal{Q}_{ \pm}$in terms of two bosonic modes $a$ and $b$ (see [48] for a general discussion on boson realizations of $s u(1,1)$ and $s u(2))$ as

$$
\mathcal{Q}_{3}=\frac{1}{2}\left(a^{\dagger} a+b^{\dagger} b+1\right), \mathcal{Q}_{+}=a^{\dagger} b^{\dagger}, \mathcal{Q}_{-}=a b,
$$


and the expression of Bargmann index- $\kappa$ basis states $|\kappa, n\rangle, n=0,1,2, \ldots, \infty$, in terms of Fock states $(|0\rangle$ denotes the Fock vacuum)

$$
\left|n_{a}\right\rangle \otimes\left|n_{b}\right\rangle=\frac{\left(a^{\dagger}\right)^{n_{a}}\left(b^{\dagger}\right)^{n_{b}}}{\sqrt{n_{a} ! n_{b} !}}|0\rangle
$$

as

$$
|\kappa, n\rangle=\frac{\left(a^{\dagger}\right)^{n}\left(b^{\dagger}\right)^{n+2 \kappa-1}}{\sqrt{n !(n+2 \kappa-1) !}}|0\rangle=\frac{\varphi_{n}\left(a^{\dagger}\right)}{\sqrt{\frac{(2 \kappa+n-1) !}{(2 \kappa-1) !}}} \frac{\varphi_{2 \kappa+n-1}\left(b^{\dagger}\right)}{\sqrt{\frac{(4 \kappa+n-2) !}{(2 \kappa-1) !}}}|0\rangle=|n\rangle_{a} \otimes|n+2 \kappa-1\rangle_{b}
$$

where we have used the monomials $\varphi_{n}$ in (31) as operator functions, since this notation will be generalized in a natural way later in eq. (67) for a Fock representation of the basis functions $\left|\begin{array}{l}j, m \\ q_{a}, q_{b}\end{array}\right\rangle$ of $\mathcal{H}_{\lambda}\left(\mathbb{D}_{4}\right)$. Note that there is always an excess of $n_{b}-n_{a}=2 \kappa-1 b$-type over $a$-type quanta, which leads to the constraint $b^{\dagger} b-a^{\dagger} a=2 \kappa-1$. The lowest weight state $|\kappa, 0\rangle=\frac{\left(b^{\dagger}\right)^{2 \kappa-1}}{\sqrt{(2 \kappa-1) !}}|0\rangle$ can be regarded as a boson condensate of $2 \kappa-1 b$-type particles, and the rest of states $|\kappa, n\rangle$ as pair $a b$ excitations ("excitons") above this condensate. The $S U(1,1)$ CS (31) can also be written as

$$
|z\rangle=\left(1-|z|^{2}\right)^{\kappa} e^{z \mathcal{Q}_{+}}|\kappa, 0\rangle
$$

Is is interesting to see that, defining $\mathcal{Z}=\left(\begin{array}{c}a^{\dagger} \\ b\end{array}\right)$ and $\mathcal{Z}^{\dagger}=\left(\begin{array}{ll}a & b^{\dagger}\end{array}\right)$, the $U(1,1)$ generators (48) can be compactly written as

$$
\mathcal{Q}_{\mu}=\frac{1}{2} \mathcal{Z}^{\dagger} \sigma_{\mu} \Gamma \mathcal{Z}
$$

with $\sigma_{\mu}=\eta_{\mu \nu} \sigma^{\nu}, \Gamma=\operatorname{diag}(-1,1), \mathcal{Q}_{ \pm}=-i \mathcal{Q}_{2} \pm \mathcal{Q}_{1}$ and the extra generator $\mathcal{Q}_{0}=$ $\frac{1}{2}\left(b^{\dagger} b-a a^{\dagger}\right)=\kappa-1$ (linear Casimir) is related to the excess of $b$ - over $a$-type quanta. Later in eq. (57) we shall relate this excess of quanta with the helicity $s=\kappa-1 / 2$ (see also Section 6 for a Lagrangian interpretation inside a twistor description of massless conformal particles). Note that for $\kappa \geq 1 / 2$ we have only positive values of the helicity $s$. Negative values of $s$ come from the alternative choice $|\kappa, 0\rangle_{-}=\frac{\left(a^{\dagger}\right)^{2 \kappa-1}}{\sqrt{(2 \kappa-1) !}}|0\rangle$ for the lowest weight state, now regarded as a boson condensate of $2 \kappa-1 a$-type particles. Actually, the quotient $U(1,1) / U(1)^{2}$ is the two-sheet hyperboloid leading to two orbits $\mathbb{D}_{1}^{ \pm}$related to positive and negative helicity $s$.

The natural (minimal) generalization of this $U(1,1)$ construction to $U(2,2)$ requires four bosonic modes $a_{1}, a_{2}, b_{1}, b_{2}$, for which Fock states are

$$
\left|n_{a}^{1}\right\rangle \otimes\left|n_{a}^{2}\right\rangle \otimes\left|n_{b}^{1}\right\rangle \otimes\left|n_{b}^{2}\right\rangle=\frac{\left(a_{1}^{\dagger}\right)^{n_{a}^{1}}\left(a_{2}^{\dagger}\right)^{n_{a}^{2}}\left(b_{1}^{\dagger}\right)^{n_{b}^{1}}\left(b_{2}^{\dagger}\right)^{n_{b}^{2}}}{\sqrt{n_{a}^{1} ! n_{a}^{2} ! n_{b}^{1} ! n_{b}^{2} !}}|0\rangle
$$

with $n_{a}^{j}, n_{b}^{j} \in \mathbb{N}$ the corresponding occupation numbers. Defining now

$$
\mathcal{Z}^{\dagger}=\left(a_{1}, a_{2}, b_{1}^{\dagger}, b_{2}^{\dagger}\right),
$$


the Jordan-Schwinger realization of the sixteen $u(2,2)$ generators in (11), compactly written as $X_{\mu \nu}=\left\{D, M_{\mu \nu}, P_{\mu}, K_{\nu}, I_{4}\right\}$ (we are adding the $4 \times 4$ identity matrix $X_{00}=I_{4}$ ), is given by

$$
\mathcal{X}_{\mu \nu}=\mathcal{Z}^{\dagger} X_{\mu \nu} \Gamma \mathcal{Z}
$$

where now $\Gamma=\operatorname{diag}(-1,-1,1,1)$. Indeed, one can easily verify that $\left[\mathcal{X}_{\mu \nu}, \mathcal{X}_{\mu^{\prime} \nu^{\prime}}\right]=$ $\mathcal{Z}^{\dagger}\left[X_{\mu \nu}, X_{\mu^{\prime} \nu^{\prime}}\right] \Gamma \mathcal{Z}$, and therefore (55) defines a (unitary) representation of $u(2,2)$ in the Fock space (53). Fixing again the excess of $b$ - over $a$-type quanta as $n_{b}^{1}+n_{b}^{2}-n_{a}^{1}-n_{a}^{2}=$ $2 \kappa-1$ [which means to fix the linear Casimir $\mathcal{X}_{00}=2 \kappa-3$, or the helicity $\mathcal{S}=\frac{1}{2} \mathcal{X}_{00}+1$ ], the basis states for fixed $\kappa$ can now be labeled in terms of three non-negative integers $\vec{n}=\left(n_{1}, n_{2}, n_{3}\right)$ as

$$
|\kappa, \vec{n}\rangle=\frac{\left(a_{1}^{\dagger}\right)^{n_{1}}\left(a_{2}^{\dagger}\right)^{n_{2}}\left(b_{1}^{\dagger}\right)^{n_{3}}\left(b_{2}^{\dagger}\right)^{2 \kappa-1+n_{1}+n_{2}+n_{3}}}{\sqrt{n_{1} ! n_{2} ! n_{3} !\left(2 \kappa-1+n_{1}+n_{2}+n_{3}\right) !}}|0\rangle, \quad n_{1}, n_{2}, n_{3}=0,1, \ldots, \infty
$$

This corresponds to the so called ladder representations of $U(2,2)$ describing massless particles with helicity $s=\kappa-\frac{1}{2}$ (see [20] for other basis and the irreducibility of this representation when restricted to the Poincaré subgroup). Indeed, if we define $\mathcal{P}^{\mu}, \mathcal{K}^{\mu}$ and $\mathcal{M}^{\mu \nu}$ the boson realization (55) of four-momentum $P^{\mu}$, 'four-acceleration' $K^{\mu}$ and Lorentz matrix $M^{\mu \nu}$ in (1), then one can verify that $\mathcal{P}^{\mu} \mathcal{P}_{\mu}=0=\mathcal{K}^{\mu} \mathcal{K}_{\mu}$ (zero mass) and

$$
\mathcal{W}^{\mu}|\kappa, \vec{n}\rangle=\left(\kappa-\frac{1}{2}\right) \mathcal{P}^{\mu}|\kappa, \vec{n}\rangle, \forall \vec{n} \in \mathbb{N}^{3}, \mu=0,1,2,3,
$$

with $\mathcal{W}_{\alpha}=\frac{i}{2} \epsilon_{\alpha \mu \nu \beta} \mathcal{M}^{\mu \nu} \mathcal{P}^{\beta}$ the Pauli-Lubanski operator and $s=\kappa-\frac{1}{2}$ the helicity.

This (Jordan-Schwinger) oscillator realization is in fact extensible to general $U(p, q)$ [22, 23] and it became popular after [24], who discussed the use of $U(6,6)$ to classify hadrons; in this case barions and antibarions belong to mutually conjugate representations with respect to $U(6)$.

This (discrete, most degenerate) representation of $U(2,2)$ is related to the quotient $\mathbb{D}_{3}=U(2,2) /[U(2,1) \times U(1)]$ (the pseudo complex projective space $\mathbb{C} P^{3}$ ) whose points $\vec{z}=\left(z_{1}, z_{2}, z_{3}\right) \in \mathbb{C}^{3}$ (in a certain patch) label the CS given by the expansion

$$
|\vec{z}\rangle=\left(1+\left|z_{1}\right|^{2}-\left|z_{2}\right|^{2}-\left|z_{3}\right|^{2}\right)^{\kappa} \sum_{n=0}^{\infty} \sum_{m=0}^{n} \sum_{l=0}^{m} \varphi_{n}^{m l}(\vec{z})|\kappa,(n-m, m-l, l)\rangle,
$$

in terms of the basis functions (56), where the coefficients

$$
\varphi_{n}^{m l}(\vec{z})=\sqrt{\left(\begin{array}{c}
2 \kappa+n-1 \\
n
\end{array}\right)\left(\begin{array}{c}
n \\
m
\end{array}\right)\left(\begin{array}{c}
m \\
l
\end{array}\right)}(-1)^{\frac{n-m}{2}} z_{1}^{n-m} z_{2}^{m-l} z_{3}^{l},
$$

are homogeneous polynomials of degree $n$ in three complex variables $\vec{z}$. The values of $z_{i}$ are not arbitrary but must fulfill $1+\left|z_{1}\right|^{2}-\left|z_{2}\right|^{2}-\left|z_{3}\right|^{2}>0$ in the present patch. These CS are normalized and also verify a resolution of the identity similar to the one in (32) but replacing the $\mathbb{D}_{1}$ integration measure by the corresponding $\mathbb{D}_{3}$ integration measure. The 
CS (58) can also be written as the exponential action [equivalent of (51)] of pair creation operators $Q_{+1}=a_{1}^{\dagger} b_{2}^{\dagger}, Q_{+2}=a_{2}^{\dagger} b_{2}^{\dagger}, Q_{+3}=b_{1}^{\dagger} b_{2}^{\dagger}$ on the ground state $|\kappa, \overrightarrow{0}\rangle=\frac{\left(b_{2}^{\dagger}\right)^{2 \kappa-1}}{\sqrt{(2 \kappa-1) !}}|0\rangle$ as

$$
|\vec{z}\rangle=\left(1+\left|z_{1}\right|^{2}-\left|z_{2}\right|^{2}-\left|z_{3}\right|^{2}\right)^{\kappa} e^{i z_{1} Q_{+1}+z_{2} Q_{+2}+z_{3} Q_{+3}}|\kappa, \overrightarrow{0}\rangle
$$

As already discussed for $U(1,1)$, again we have two conformal orbits $\mathbb{D}_{3}^{ \pm}$corresponding to phase-spaces of negative and positive helicity zero-mass particles. The ground state for negative helicity particles can be namely taken as $|\kappa, \overrightarrow{0}\rangle_{-}=\frac{\left(a_{1}^{\dagger}\right)^{2 \kappa-1}}{\sqrt{(2 \kappa-1) !}}|0\rangle$, where there is an excess of $2 \kappa-1 a$-type over $b$-type quanta.

However, these are still not the CS (28) we are dealing with in this article. Actually, the CS (28) will be related to massive conformal particles (see later in Sec. 6 for a Lagrangian picture). The question is: is there a boson realization like (58) and (60) but for the CS (28) labeled by points $Z$ in the Cartan domain $\mathbb{D}_{4}$ ?. The answer is positive and it will be given later in Proposition 5.2. Indeed, there is another way of extending the $U(1,1)$ construction to $U(2,2)$ by defining now

$$
\mathcal{Z}=\left(\begin{array}{c}
\mathbf{a}^{\dagger} \\
\mathbf{b}
\end{array}\right)=\left(\begin{array}{ll}
a_{0}^{\dagger} & a_{2}^{\dagger} \\
a_{1}^{\dagger} & a_{3}^{\dagger} \\
b_{0} & b_{1} \\
b_{2} & b_{3}
\end{array}\right)
$$

which can be seen as a "compound" $\mathcal{Z}=\left(\mathcal{Z}_{1}, \mathcal{Z}_{2}\right)$ of two zero-mass systems $\mathcal{Z}_{1}=$ $\left(a_{0}^{\dagger}, a_{1}^{\dagger}, b_{0}, b_{2}\right)^{t}$ and $\mathcal{Z}_{2}=\left(a_{2}^{\dagger}, a_{3}^{\dagger}, b_{1}, b_{3}\right)^{t}$ with certain constraints given below (see later in this Section for an explicit proof). The new oscillator realization

$$
\mathcal{X}_{\mu \nu}=\operatorname{tr}\left(\mathcal{Z}^{\dagger} X_{\mu \nu} \Gamma \mathcal{Z}\right)
$$

of the sixteen $u(2,2)$ matrix generators $X_{\mu \nu}$ in (1) [plus identity $X_{00}$ ], defines a (unitary) representation of $u(2,2)$ in the Fock space with basis states

$$
\left|\mathbf{n}_{a}\right\rangle \otimes\left|\mathbf{n}_{b}\right\rangle=\left|\begin{array}{cc}
n_{a}^{0} & n_{a}^{1} \\
n_{a}^{2} & n_{a}^{3}
\end{array}\right\rangle \otimes\left|\begin{array}{cc}
n_{b}^{0} & n_{b}^{1} \\
n_{b}^{2} & n_{b}^{3}
\end{array}\right\rangle=\prod_{\mu=0}^{3} \frac{\left(a_{\mu}^{\dagger}\right)^{n_{a}^{\mu}}\left(b_{\mu}^{\dagger}\right)^{n_{b}^{\mu}}}{\sqrt{n_{a}^{\mu} ! n_{b}^{\mu} !}}|0\rangle .
$$

Let us look for the expression of the basis states $\left|\begin{array}{l}j, m \\ q_{a}, q_{b}\end{array}\right\rangle$ in (26) in terms of the Fock basis (63). The $U(2,2)$ analogue of the $U(1,1)$ (linear Casimir) constraint $2 \mathcal{Q}_{0}=b^{\dagger} b-a a^{\dagger}=$ $2(\kappa-1)$ [remember (52)] on the basis states $|\kappa, n\rangle(\underline{50})$, here adopts the matrix form

$$
\mathcal{Z}^{\dagger} \Gamma \mathcal{Z}=\mathbf{b}^{\dagger} \mathbf{b}-\mathbf{a a}^{\dagger}=(\lambda-4) \mathcal{I}_{2}
$$

on the basis states $\left|\begin{array}{l}j, m \\ q_{a}, q_{b}\end{array}\right\rangle$, where $\mathcal{I}_{2}$ denotes the $2 \times 2$ identity operator. In particular, there is an excess of $\sum_{\mu=0}^{3} n_{b}^{\mu}-n_{a}^{\mu}=2(\lambda-2)$ of $b$ - over $a$-type quanta, that is, the linear Casimir operator $\mathcal{X}_{00}=\sum_{\mu=0}^{3} b_{\mu}^{\dagger} b_{\mu}-a_{\mu} a_{\mu}^{\dagger}$ is fixed to $2(\lambda-4)$. From (37), we also see that the dilation operator $\mathcal{D}=2+\frac{1}{2} \sum_{\mu=0}^{3}\left(a_{\mu}^{\dagger} a_{\mu}+b_{\mu}^{\dagger} b_{\mu}\right)$ provides the relation

$$
2+\frac{1}{2} \sum_{\mu=0}^{3}\left(n_{a}^{\mu}+n_{b}^{\mu}\right)=2 j+2 m+\lambda
$$


with $2 j+2 m$ the homogeneity degree of $\varphi_{q_{a}, q_{b}}^{j, m}$, which also coincides with the total number of pair $a b$ excitations ("excitons") over the lowest-weight (zero homogeneity degree) ground state $\left.\left.\left|\varphi_{0}\right\rangle \equiv\right|_{q_{a}=0, q_{b}=0} ^{j=0, m=0}\right\rangle$, which is made of $2(\lambda-2)$ b-type quanta and can expressed in terms of Fock states as:

$$
\left|\varphi_{0}\right\rangle=\frac{\operatorname{det}\left(\mathbf{b}^{\dagger}\right)^{\lambda-2}}{(\lambda-2) ! \sqrt{\lambda-1}}|0\rangle=\left|\begin{array}{ll}
0 & 0 \\
0 & 0
\end{array}\right\rangle_{a} \otimes \sum_{k=0}^{\lambda-2} \frac{(-1)^{k}}{\sqrt{\lambda-1}}\left|\begin{array}{cc}
\lambda-2-k & k \\
k & \lambda-2-k
\end{array}\right\rangle_{b} .
$$

Indeed, one can easily check that $\left|\varphi_{0}\right\rangle$ fulfills the constraints $\mathcal{Z}^{\dagger} \Gamma \mathcal{Z}=(\lambda-4) \mathcal{I}_{2}$. In order to obtain the expression of the rest of basis states $\left|q_{q_{a}, q_{b}}^{j, m}\right\rangle$ in terms of Fock states (63), we have firstly made use of the differential representation $\mathfrak{X}_{\mu \nu}$ of the bosonic operators $\mathcal{X}_{\mu \nu}$ and applying, step by step*, ladder operators (38,39,42) and (43) to the lowest-weight state (66), we have finally arrived to the expression

$$
\left.||_{q_{a}, q_{b}}^{j, m}\right\rangle=\frac{1}{\sqrt{2 j+1}} \sum_{q=-j}^{j} \frac{\varphi_{q_{a}, q}^{j, m}\left(\mathbf{a}^{\dagger}\right)}{\sqrt{\frac{(\lambda-2) !(\lambda-1) !}{(\lambda+2 j+m-1) !(\lambda+m-2) !}}} \frac{\varphi_{q, q_{b}}^{j, \lambda+m-2}\left(\mathbf{b}^{\dagger}\right)}{\sqrt{\frac{(\lambda-2) !(\lambda-1) !}{(2 \lambda+2 j+m-3) !(2 \lambda+m-4) !}}}|0\rangle,
$$

where we are now treating the homogeneous polynomials $\varphi_{q, q^{\prime}}^{j, m}$ in (23) as operator functions, since there is not ordering problem (all $a_{\mu}^{\dagger}$ and $b_{\mu}^{\dagger}$ commute). This is the $S U(2,2)$ version of eq. (50) for the Bargmann index- $\kappa$ basis states $|\kappa, n\rangle$ of $S U(1,1)$, with the role of $\kappa$ played now by $\lambda$ and the role of the monomials $\varphi_{n}(z)$ played now by the homogeneous polynomials $\varphi_{q_{a}, q_{b}}^{j, m}(Z)$.

In the process we have found extra restrictions to the number $n_{a}^{\mu}$ and $n_{b}^{\mu}$ of $a$ - and b-type bosons like:

$$
\left(n_{b}^{0}+n_{b}^{2}\right)-\left(n_{a}^{0}+n_{a}^{1}\right)=\lambda-2=\left(n_{b}^{1}+n_{b}^{3}\right)-\left(n_{a}^{2}+n_{a}^{3}\right)
$$

and

$$
\begin{aligned}
n_{a}^{0}-n_{a}^{1}+n_{a}^{2}-n_{a}^{3} & =2 q_{a}, \\
n_{b}^{0}+n_{b}^{1}-n_{b}^{2}-n_{b}^{3} & =2 q_{b} .
\end{aligned}
$$

The restrictions (69) say that the third angular momentum components $q_{a}$ and $q_{b}$, measure the imbalance between $\mu=\{0,2\}$ (spin up) and $\mu=\{1,3\}$ (spin down) $a$-type bosons and $\mu=\{0,1\}$ (spin up) and $\mu=\{2,3\}$ (spin down) $b$-type bosons.

The restriction (68) could be interpreted by saying that both zero-mass particles, $\mathcal{Z}_{1}$ and $\mathcal{Z}_{2}$ forming the compound $\mathcal{Z}=\left(\mathcal{Z}_{1}, \mathcal{Z}_{2}\right)$, carry helicity $s=(\lambda-2) / 2$. Indeed, if we define the four-momentum $\mathcal{P}_{p}^{\mu}$, Lorentz $\mathcal{M}_{p}^{\mu \nu}$ and Pauli-Lubanski $\mathcal{W}_{p}^{\mu}$ operators for each separated particle $\mathcal{Z}_{p}\left[\mathcal{Z}_{1}=\left(a_{0}^{\dagger}, a_{1}^{\dagger}, b_{0}, b_{2}\right)^{t}\right.$ and $\left.\mathcal{Z}_{2}=\left(a_{2}^{\dagger}, a_{3}^{\dagger}, b_{1}, b_{3}\right)^{t}\right]$ of the compound $\mathcal{Z}=\left(\mathcal{Z}_{1}, \mathcal{Z}_{2}\right)$ as

$$
\mathcal{P}_{p}^{\mu}=\mathcal{Z}_{p}^{\dagger} P^{\mu} \Gamma \mathcal{Z}_{p}, \quad \mathcal{M}_{p}^{\mu \nu}=\mathcal{Z}_{p}^{\dagger} M^{\mu \nu} \Gamma \mathcal{Z}_{p}, p=1,2, \quad \mathcal{W}_{\alpha}^{p}=\frac{i}{2} \epsilon_{\alpha \mu \nu \beta} \mathcal{M}_{p}^{\mu \nu} \mathcal{P}_{p}^{\beta}, p=1,2,
$$

${ }^{*}$ We do not present here the (rather cumbersome) steps to get this result. We must acknowledge the benefits of Mathematica add-on packages like "Quantum Algebra" to check this and some other expressions along this Section. These packages are available at [49]. 
then one can check, for each individual particle $p=1,2$, that

$$
\left.\left.\mathcal{P}_{p}^{\mu} \mathcal{P}_{p \mu}=0,\left.\mathcal{W}_{p}^{\mu}\right|_{q_{a}, q_{b}} ^{j, m}\right\rangle=\left.\frac{\lambda-2}{2} \mathcal{P}_{p}^{\mu}\right|_{q_{a}, q_{b}} ^{j, m}\right\rangle, p=1,2
$$

are valid for any basis vector $\left|\begin{array}{l}j, m \\ q_{a}, q_{b}\end{array}\right\rangle$, which means that both particles are massless and carry helicity $s_{p}=(\lambda-2) / 2, p=1,2$. However, the compound $\mathcal{Z}=\left(\mathcal{Z}_{1}, \mathcal{Z}_{2}\right)$ displays a continuum mass spectrum since

$$
\left.\left.\mathcal{P}^{\mu} \mathcal{P}_{\mu}||_{q_{a}, q_{b}}^{j, m}\right\rangle=\left.4 \sqrt{m(2 j+m+1)(\lambda+m-2)(\lambda+2 j+m-1)}\right|_{q_{a}, q_{b}} ^{j, m-1}\right\rangle,
$$

with $\mathcal{P}^{\mu}=\operatorname{tr}\left(\mathcal{Z}^{\dagger} P^{\mu} \Gamma \mathcal{Z}\right)$ the compound's four-momentum, and the corresponding PauliLubanski operator is zero $\left.\left.\mathcal{W}^{\mu}\right|_{q_{a}, q_{b}} ^{j, m}\right\rangle=0$, since the compound $\left(\mathcal{Z}_{1}, \mathcal{Z}_{2}\right)$ is spin-less (see e.g. [29] for more general, spinning, representations of the conformal group in a geometrical setting).

The existence of a continuum mass spectrum was already adverted for the differential representation in (43). The fact that two massless particles can form a massive compound can result somehow awkward. In the Standard Model, particles acquire mass from the Higgs boson trough the "spontaneous breakdown" of the gauge symmetry. However, here we see that coupling massless particles can also result in a massive compound. This seems to be a profound result that deserves more attention and will be studied elsewhere.

We shall now study the quantum statistics of the compound under the exchange $\left(\mathcal{Z}_{1}, \mathcal{Z}_{2}\right) \rightarrow\left(\mathcal{Z}_{2}, \mathcal{Z}_{1}\right)$ of its two massless constituents for a given $\lambda$. The result is given in the next Theorem.

Theorem 5.1. Wave functions for the compound $\mathcal{Z}=\left(\mathcal{Z}_{1}, \mathcal{Z}_{2}\right)$ are symmetric under the exchange $\left(\mathcal{Z}_{1}, \mathcal{Z}_{2}\right) \rightarrow\left(\mathcal{Z}_{2}, \mathcal{Z}_{1}\right)$ for $\lambda$ even and antisymmetric for $\lambda$ odd. In particular, the basis functions (67) verify

$$
\widetilde{\left|q_{q_{a}, q_{b}}^{j, m}\right\rangle}=(-1)^{\lambda}\left|\begin{array}{l}
j, m \\
q_{a}, q_{b}
\end{array}\right\rangle
$$

where $\widetilde{\mid \begin{array}{l}j, m \\ q_{a}, q_{b}\end{array}}$ is constructed as in (67) but replacing

$$
\mathbf{a}=\left(\begin{array}{ll}
a_{0} & a_{1} \\
a_{2} & a_{3}
\end{array}\right) \rightarrow \widetilde{\mathbf{a}}=\left(\begin{array}{ll}
a_{2} & a_{3} \\
a_{0} & a_{1}
\end{array}\right), \mathbf{b}=\left(\begin{array}{ll}
b_{0} & b_{1} \\
b_{2} & b_{3}
\end{array}\right) \rightarrow \widetilde{\mathbf{b}}=\left(\begin{array}{ll}
b_{1} & b_{0} \\
b_{3} & b_{2}
\end{array}\right),
$$

that is, exchange of rows in a-type particles and exchange of columns in b-type particles. Proof: The proof is simple when one realizes that the operator functions (23) verify

$$
\varphi_{q_{a}, q_{b}}^{j, m}\left(\widetilde{\mathbf{b}}^{\dagger}\right)=(-1)^{m} \varphi_{-q_{a}, q_{b}}^{j, m}\left(\mathbf{b}^{\dagger}\right), \quad \varphi_{q_{a}, q_{b}}^{j, m}\left(\widetilde{\mathbf{a}}^{\dagger}\right)=(-1)^{m} \varphi_{q_{a},-q_{b}}^{j, m}\left(\mathbf{a}^{\dagger}\right) .
$$

Taking into account that $(-1)^{2 q}=(-1)^{2 j}$ for any $q=-j, \ldots, j$ and doing some algebraic manipulations, one arrives to the identity (73).

This is a consequence of the indistinguishability of the two zero-mass particles, $\mathcal{Z}_{1}$ and $\mathcal{Z}_{2}$, forming the compound $\mathcal{Z}=\left(\mathcal{Z}_{1}, \mathcal{Z}_{2}\right)$. The even/odd character of $\lambda$ (related to the helicity $(\lambda-2) / 2$ of the massless particles and to the excess number $2(\lambda-2)$ of unpaired particles) determines the bosonic/fermionic character of the compound. 
Note that the two massless particles of the compound are correlated (constrained) and identical. These correlations are worth studying more carefully and will be left for future work [50].

Before finishing this Section, we shall provide a boson realization like (51) but for the CS (28) labeled by points $Z$ in the complex domain $\mathbb{D}_{4}$.

Proposition 5.2. Let us denote by $\mathcal{K} \equiv \mathcal{K}^{\mu} \sigma_{\mu}=-2 \mathbf{a}^{\dagger} \mathbf{b}^{\dagger}$, with $\mathcal{K}^{\mu}=\operatorname{tr}\left(\mathcal{Z}^{\dagger} K^{\mu} \Gamma \mathcal{Z}\right)$ the oscillator realization of the matrix $K^{\mu}$ in (11). The $C S|Z\rangle$ in (28) can be written as the exponential action of creation particle-hole operators $\mathcal{K}$ on the lowest-weight state $\left|\varphi_{0}\right\rangle$ as

$$
|Z\rangle=\operatorname{det}\left(\sigma^{0}-Z^{\dagger} Z\right)^{\lambda / 2} e^{-\frac{1}{2} \operatorname{tr}\left(Z^{t} \mathcal{K}\right)}\left|\varphi_{0}\right\rangle .
$$

Proof: Proving (74) is equivalent to prove that

$$
\left.e^{-\mathcal{A}}\left|\varphi_{0}\right\rangle=\left.\sum_{m=0}^{\infty} \sum_{j \in \mathbb{N} / 2} \sum_{q_{a}, q_{b}=-j}^{j} \varphi_{q_{a}, q_{b}}^{j, m}(Z)\right|_{q_{a}, q_{b}} ^{j, m}\right\rangle,
$$

with

$$
\mathcal{A} \equiv \frac{1}{2} \operatorname{tr}\left(Z^{t} \mathcal{K}\right)=(-1)^{\mu} z_{\mu} \mathcal{K}^{\mu},(\text { sum on } \mu) .
$$

Note that the equivalence of the expressions (28) and (74) for CS on $U(2,2) / U(2)^{2}$ is the matrix counterpart of the (easier to prove) equivalence of (31) and (51) for CS on $U(1,1) / U(1)^{2}$. To prove (75) we shall proceed by induction on the homogeneity degree in $Z$. More precisely, we shall firstly prove that the identity

$$
\left.\frac{(-\mathcal{A})^{n}}{n !}\left|\varphi_{0}\right\rangle=\left.\sum_{j=\frac{\text { odd }(n)}{2}}^{n-\frac{\text { odd }(n)}{2}} \sum_{q_{a}=-j}^{j} \sum_{q_{b}=-j}^{j} \varphi_{q_{a}, q_{b}}^{j, \frac{n}{2}-j}(Z)\right|_{q_{a}, q_{b}} ^{j, \frac{n}{2}-j}\right\rangle,
$$

is true for any $n \in \mathbb{N}$, where odd $(n)=\left(1-(-1)^{n}\right) / 2$. In fact, it is trivially fulfilled for $n=0$. For $n=1$ we have that

$$
\begin{aligned}
-\mathcal{A}\left|\varphi_{0}\right\rangle= & \left.\left.-\sqrt{\lambda}\left(\left.\left(z_{3}-z_{0}\right)\right|_{\frac{-1}{2}, \frac{1}{2}} ^{\frac{1}{2}, 0}\right\rangle-\left.\left(z_{3}+z_{0}\right)\right|_{\frac{1}{2}, \frac{1}{2}} ^{\frac{1}{2}, 0}\right\rangle-\left.\left(z_{1}-i z_{2}\right)\right|_{\frac{1}{2}, \frac{-1}{2}} ^{\frac{1}{2}, 0}\right\rangle \\
& \left.\left.\left.-\left.\left(z_{1}+i z_{2}\right)\right|_{\frac{1}{2}, \frac{1}{2}, 0} ^{\mid \frac{1}{2}}\right\rangle\right)=\left.\sum_{q_{a}, q_{b}=-1 / 2}^{1 / 2} \varphi_{q_{a}, q_{b}}^{\frac{1}{2}, 0}(Z)\right|_{q_{a}, q_{b}} ^{\frac{1}{2}, 0}\right\rangle,
\end{aligned}
$$

where we have made use of the differential representation $\mathfrak{K}^{\mu}$ of $\mathcal{K}^{\mu}$ in (39) and the definition of $\varphi_{q_{a}, q_{b}}^{j, m}(Z)$ in (23). Therefore, the hypothesis (77) is true for $n=1$. Assuming (77) to be true for a given $n$, and using again (39) and the definition (23), we finally arrive 
to

$$
\begin{aligned}
\frac{(-\mathcal{A})^{n+1}}{(n+1) !}\left|\varphi_{0}\right\rangle & \left.=\left.\frac{-1}{n+1} \sum_{j=\frac{\text { odd }(n)}{2}}^{n-\frac{\text { odd }(n)}{2}} \sum_{q_{a}=-j}^{j} \sum_{q_{b}=-j}^{j} \varphi_{q_{a}, q_{b}}^{j, \frac{n}{2}-j}(Z) \mathcal{A}\right|_{q_{a}, q_{b}} ^{j, \frac{n}{2}-j}\right\rangle \\
& \left.=\left.\sum_{j=\frac{\operatorname{odd}(n+1)}{2}}^{n+1-\frac{\operatorname{odd}(n+1)}{2}} \sum_{q_{a}=-j}^{j} \sum_{q_{b}=-j}^{j} \varphi_{q_{a}, q_{b}}^{j, \frac{n+1}{2}-j}(Z)\right|_{q_{a}, \frac{n+1}{2} q_{b}} ^{j, j}\right\rangle,
\end{aligned}
$$

which states that the hypothesis (77) is true for $n+1$, thus completing the proof by induction. The expansion of the exponential $e^{-\mathcal{A}}=\sum_{n=0}^{\infty} \frac{(-\mathcal{A})^{n}}{n !}$ completes the proof of (75) and then of (74)

The operator $\mathcal{K}^{\mu}=-\operatorname{tr}\left(\mathbf{a}^{\dagger} \mathbf{b}^{\dagger} \sigma_{\mu}\right)$ creates particle-hole pairs (excitons) and $\mathcal{P}^{\mu}=$ $-\operatorname{tr}\left(\mathbf{b} \mathbf{a} \sigma^{\mu}\right)$ annihilates excitons. It is said in the literature that excitons are almost bosons [51] since exciton creation and annihilation operators fulfill bosonic commutation relations plus corrections in the number of particle-hole pairs which arise from the interaction between excitons. In our case, if we renormalize the two-body creation $\mathcal{K}_{\mu} / \sqrt{2(\lambda-2)} \equiv \mathcal{E}_{\mu}^{\dagger}$ and annihilation $\mathcal{P}_{\mu} / \sqrt{2(\lambda-2)} \equiv \mathcal{E}_{\mu}$ operators with the square root of the number $2(\lambda-2)$

of unpaired particles, and we use the fact that $\left|\begin{array}{l}j, m \\ q_{a}, q_{b}\end{array}\right\rangle$ are eigenstates of $\mathcal{D}$ with eigenvalue $n_{e}+\lambda\left[n_{e}=2 j+2 m\right.$ is the number of excitons; remember (37,65) $]$, then the basic commutator $\left[\mathcal{K}_{\mu}, \mathcal{P}_{\nu}\right]=2\left(\eta_{\mu \nu} \mathcal{D}+\mathcal{M}_{\mu \nu}\right)$ says that (we restrict ourselves to $\mu=\nu$ for simplicity)

$$
\left\langle q_{a},\left.q_{b}\left|\left[\mathcal{E}_{\mu}^{\dagger}, \mathcal{E}_{\mu}\right]\right|\right|_{q_{a}, q_{b}} ^{j, m}\right\rangle=2 \eta_{\mu \mu} \frac{\lambda+n_{e}}{2(\lambda-2)} \simeq \eta_{\mu \mu}\left(1+O\left(\frac{n_{e}}{\lambda}\right)\right)
$$

for a large number of unpaired particles, $\lambda \gg 1$. This result agrees with the general fact that excitons are almost bosons as long as $n_{e} \ll \lambda$ [note that the commutator for the temporal $\mu=0$ component has the reversed desired sign].

The expression (74) shows the coherent state $|Z\rangle$ as a "Bose-Einstein condensate" of excitons. Interesting physical phenomena of Bose-Einstein condensation of excitons and biexcitons can be found in [52]. We believe that our abstract construction of coherent states of excitons can provide an interesting framework to study physical applications in this context.

\section{Lagrangian picture and physical interpretations}

Let us propose a physical interpretation of the previous abstract mathematical construction by making use of the twistor particle picture (see e.g. [37, 38, 39, 40, 41]) and $U(2,2)$ nonlinear sigma model Lagrangians (see e.g. [29, 53, 54]). General integrable Hamiltonian systems on $S U(2,2)$ and $S U(p, q)$ have also been discussed in [55, 56].

The twistor space $\mathbb{T}$ is a 4-dimensional complex vector space $\mathbb{C}^{4}$ with points $\zeta=$ $\left(\alpha_{1}, \alpha_{2}, \beta_{1}, \beta_{2}\right)^{t} \in \mathbb{C}^{4}(t$ means transpose $)$, and their duals $\zeta^{\dagger}=\left(\bar{\alpha}_{1}, \bar{\alpha}_{2}, \bar{\beta}_{1}, \bar{\beta}_{2}\right)$. The bilinear product (related to the helicity) $\varsigma=\zeta^{\dagger} \Gamma \zeta=\left|\beta_{1}\right|^{2}+\left|\beta_{2}\right|^{2}-\left|\alpha_{1}\right|^{2}-\left|\alpha_{2}\right|^{2}$ is invariant 
under the natural linear action of $U(2,2)$ in (2). It measures the imbalance between, let us say, "particle" ( $\beta$ spinor) and "antiparticle" ( $\alpha$ spinor $)$, which transform under conjugated $U(2)$ transformations, $U(2)_{\alpha} \times U(2)_{\beta} \subset U(2,2)$. It is well known that twistors describe massless particles. The phase space of a massless conformal particle is the pseudo complex projective space $\mathbb{D}_{3}=U(2,2) /[U(2,1) \times U(1)]$, which carries two open orbits $\mathbb{D}_{3}^{ \pm}$ corresponding to the sign of the helicity. We shall take representatives $\zeta \in \mathbb{D}_{3}^{ \pm}$normalized according to $\varsigma=\zeta^{\dagger} \Gamma \zeta= \pm 1$. Note that the first two columns of $g \in U(2,2)$ in eq. (2) belong to $\mathbb{D}_{3}^{-}$and the last two ones belong to $\mathbb{D}_{3}^{+}$. A Lagrangian whose quantization leads to the oscillator realization discussed in (55) and (56) is formulated in terms of a nonlinear $\sigma$-model as follows. Although we are in a quantum mechanical setting (finite number of particles), we shall consider the general many particle case (field theory) to which the former case reduces for zero spatial dimensions. Therefore, consider the twistor $\zeta$ as a field on spacetime $\zeta\left(x^{\mu}\right)$ [for a single particle, we would consider a function of just time $\left.\zeta\left(x^{0}\right)\right]$ and denote $\partial_{\mu} \zeta=\partial \zeta / \partial x^{\mu}$ [resp. $\dot{\zeta}=d \zeta / d x^{0}$ for mechanics]. In $\mathbb{D}_{3}$, the twistors $\zeta$ and $\zeta u$, with $u \in U(1)$ an arbitrary phase, are equivalent. Therefore, $\zeta$ transforms as $\zeta \rightarrow g \zeta u$ under global $g \in U(2,2)$ and local $u \in U(1)$. The Lagrangian $L=\frac{1}{2} \partial_{\mu} \zeta^{\dagger} \Gamma \partial^{\mu} \zeta$ is not $U(1)$ gauge invariant, but the minimal coupling $\partial_{\mu} \rightarrow D_{\mu}-A_{\mu}$, with $U(1)$-gauge field $A_{\mu}=\varsigma \zeta^{\dagger} \Gamma \partial_{\mu} \zeta$, renders the Lagrangian

$$
L_{\varsigma}=\frac{1}{2} \partial_{\mu} \zeta^{\dagger}\left(1-\varsigma \Gamma \zeta \zeta^{\dagger}\right) \Gamma \partial^{\mu} \zeta
$$

$U(1)$-gauge invariant in each orbit $\mathbb{D}_{3}^{\varsigma}$ with $\varsigma= \pm 1$ and $\left(1-\varsigma \Gamma \zeta \zeta^{\dagger}\right) \Gamma$ a projector. Other gauge invariant terms linear in "velocity" can be added to the Lagrangian in the quantum mechanical case [29]. In the quantization process, the negative-energy problem is overcome by assigning $\zeta \rightarrow \mathcal{Z}$, with $\mathcal{Z}$ the operator-valued twistor in (54); that is, we assign annihilation operators $\left(b_{1}, b_{2}\right)$ to particles $\beta$ and creation operators $\left(a_{1}^{\dagger}, a_{2}^{\dagger}\right)$ to antiparticles $\alpha$. This reminds the negative-energy problem for Dirac particles, although the solution there entails the imposition of anticommutation relations. The quantum mechanical helicity $\mathcal{S}=\frac{1}{2} \mathcal{Z}^{\dagger} \Gamma \mathcal{Z}+1$ can take any half-integer value.

Let us now discuss the more involved massive case. A single massive particle turns out to be described in terms of two (or more) twistors $\zeta_{1}, \zeta_{2} \in \mathbb{C}^{4}$ arranged as:

$$
\mathbf{Z}=\left(\zeta_{1}, \zeta_{2}\right)=\left(\begin{array}{ll}
\alpha_{11} & \alpha_{12} \\
\alpha_{21} & \alpha_{22} \\
\beta_{11} & \beta_{12} \\
\beta_{21} & \beta_{22}
\end{array}\right)
$$

The (massless) constraint $\varsigma=\zeta^{\dagger} \Gamma \zeta= \pm 1$ now adopts the form $\Sigma=\mathbf{Z}^{\dagger} \Gamma \mathbf{Z}= \pm I_{2}$ (with $I_{2}$ the $2 \times 2$ identity matrix). The phase space of a massive conformal particle is the complex pseudo-Grassmann manifold $\mathbb{D}_{4}=U(2,2) /[U(2) \times U(2)]$ of 2-planes in $\mathbb{C}^{4}$, which carries two open orbits $\mathbb{D}_{4}^{ \pm}$corresponding to the $\operatorname{sign} \varsigma= \pm 1$ of $\Sigma=\varsigma I_{2}$. Note that the first block-(two)-column of $g \in U(2,2)$ in eq. (2) belongs to $\mathbb{D}_{4}^{-}$and the last one belongs to $\mathbb{D}_{4}^{+}$. In $\mathbb{D}_{4}$, the bi-twistors $\mathbf{Z}$ and $\mathbf{Z} U$, with $U \in U(2)$ an arbitrary rotation, 
are equivalent. Therefore, $\mathbf{Z}$ transforms as $\mathbf{Z} \rightarrow g \mathbf{Z} U$ under global $g \in U(2,2)$ and local $U \in U(2)$. Minimal coupling $\partial_{\mu} \rightarrow D_{\mu}-A_{\mu}$, with $U(2)$-gauge field $A_{\mu}=\varsigma \mathbf{Z}^{\dagger} \Gamma \partial_{\mu} \mathbf{Z}$, renders the Lagrangian

$$
L_{\varsigma}=\frac{1}{2} \operatorname{tr}\left[\partial_{\mu} \mathbf{Z}^{\dagger}\left(1-\varsigma \Gamma \mathbf{Z} \mathbf{Z}^{\dagger}\right) \Gamma \partial^{\mu} \mathbf{Z}\right]
$$

$U(2)$-gauge invariant in each orbit $\mathbb{D}_{4}^{\varsigma}$, with $\left(1-\varsigma \Gamma \mathbf{Z} \mathbf{Z}^{\dagger}\right) \Gamma$ a projector. One can eliminate four complex fields (out of the original eight) from the theory by making a choice of gauge

$$
\mathbf{Z}(Z)=\left(\begin{array}{c}
Z \Delta_{2} \\
\Delta_{2}
\end{array}\right)
$$

which leaves four complex degrees of freedom $Z$ parametrizing a point on $\mathbb{D}_{4}$ in (7), with $\Delta_{2}$ defined in (4) (note that this choice corresponds to $\varsigma=1$ ). This factorization has to do with the Iwasawa decomposition (11).

In the quantization process, the negative-energy problem is now overcome by assigning $\mathbf{Z} \rightarrow \mathcal{Z}$, with $\mathcal{Z}$ the operator-valued bi-twistor in (61); that is,

$$
\left(\begin{array}{ll}
\alpha_{11} & \alpha_{12} \\
\alpha_{21} & \alpha_{22}
\end{array}\right) \rightarrow\left(\begin{array}{cc}
a_{0}^{\dagger} & a_{2}^{\dagger} \\
a_{1}^{\dagger} & a_{3}^{\dagger}
\end{array}\right),\left(\begin{array}{ll}
\beta_{11} & \beta_{12} \\
\beta_{21} & \beta_{22}
\end{array}\right) \rightarrow\left(\begin{array}{ll}
b_{0} & b_{1} \\
b_{2} & b_{3}
\end{array}\right) .
$$

The lowest weight state $\left|\varphi_{0}\right\rangle$ in (66) can be regarded as a boson condensate of $2(\lambda-2)$ $b$-type quanta (let's say, "matter" or "particles"), and the rest of states $\left|q_{a}, q_{b}\right\rangle$ as pair $a b$ ("matter-antimatter" or "particle-hole") excitations ("excitons", in condensed matter jargon) above this condensate. Another possibility consists of considering an excess of $a$-type quanta over $b$-type quanta. This particle excess (or, more precisely, $\lambda$ ) determines the statistics (either bosonic or fermionic) of the physical states under the interchange of the two particles of the compound [interchange of columns in (61) or in (81)] according to (73). One would be tempted to relate this asymmetry with the parity violation in weak nuclear forces or, even further, with the matter-antimatter asymmetry in the universe; but, for the time being, these are just simple speculations.

We believe that our construction will be useful not only for the better understanding of the structure of conformal quantum particles, but also for general pairing systems like nuclear pairing phenomenon or superconductivity in solid state physics. The quantum number $\lambda$ in these models would be related to the so called "seniority number", which counts the number of unpaired nucleons or electrons in each case. We must say that the infinite-dimensional character of the $U(2,2)$ [or general $U(N, N)$ ] bosonic representation should be in principle more appropriate to describe superconductivity phenomena, where the number of free electrons is very large, although projection techniques on finite number of particles could also be applied. In fact, from the oscillator realization of (a certain number of copies of $) s u(2)$ and $s u(1,1)$, many interesting pairing many-body Hamiltonians have been built like Bardeen-Cooper-Schrieffer, Lipkin-Meshkov-Glick, etc (see e.g. [57, 58 in the context of Richardson-Gaudin models). The construction made here for $U(2,2)$ can be easily generalized to $U(N, N)$ following the guidelines of the extended MacMahonSchwinger master theorem proved by us in [28]; this also opens the possibility of dealing with even more complex situations (more degrees of freedom). 
The Euclidean $[\Gamma=\operatorname{diag}(1,1,1,1) \rightarrow U(4)]$ version of the present construction has been recently studied in [59], where a physical interpretation inside the bilayer fractional quantum Hall effect has been put forward. In particular, $a$ and $b$ correspond to the top and bottom layers (the pseudospin) and the index $\lambda$ is related to the number of flux quanta bound to a bi-fermion in the composite fermion picture of Jain for fractions of the filling factor $\nu=2$. We recommend the interested reader to have a look at this Euclidean $U(4)$ version to perceive similarities and differences with the present $U(2,2)$ construction.

\section{Acknowledgements}

Work partially supported by the Spanish MICINN, University of Granada and Junta de Andalucía under projects FIS2011-29813-C02-01, PP2012-PI04 and FQM1861 respectively.

\section{References}

[1] E. L. Hill, On accelerated coordinate systems in classical and relativistic mechanics, Phys. Rev. 67 (1945) 358-363.

[2] L. J. Boya and J. M. Cerveró, Contact Transformations and Conformal Group. I. Relativistic Theory, Int. J. Theor. Phys. 12 (1975) 47-54

[3] T. Fulton, F. Rohrlich and L. Witten, Physical consequences to a coordinate transformation to a uniformly accelerating frame, Nuovo Cimento 26 (1962) 652-671

[4] E.L. Hill, On a Type of Kinematical "Red-Shift" Phys. Rev. 68 (1945) 232.

[5] H. Weyl, Space, Time and Matter, Dover. NY. First Edition 1922

[6] H.A. Kastrup, Gauge properties of the Minkowski space, Phys. Rev. 150, 1183 (1966)

[7] P. Jordan, Z. Phys. 94 (1935) 531

[8] L.C. Biedenharn, J.D. Louck, Angular Momentum in Quantum Physics, AddisonWesley, Reading, MA, 1981;

L.C. Biedenharn, J.D. Louck, The Racah-Wigner Algebra in Quantum Theory, Addison-Wesley, New York, MA 1981

[9] J. Schwinger, "On angular momentum", in Quantum Theory of Angular Momentum, edited by L. Biedenharn and H. Van Dam (Academic, New York, 1965).

[10] M. Moshinsky and J.G. Nagel, Complete classification of states of supermultiplet theory, Phys. Lett. 5 (1963) 173-174

[11] M. Moshinsky, The harmonic oscillator and supermultiplet theory: (I) The single shell picture, Nucl. Phys. 31 (1962) 384 
[12] M. Moshinsky, Bases for the Irreducible Representations of the Unitary Groups and Some Applications, J. Math. Phys. 4, 1128 (1963)

[13] M. Moshinsky, Group Theory and the Many-Body Problem (Gordon \& Breach, New York, 1968).

[14] F. Iachello and R. D. Levine. Algebraic Theory of Molecules. Oxford University Press, Oxford, 1995.

[15] F. Iachello and A. Arima. The Interacting Boson Model. Cambridge University Press, Cambridge, 1987.

[16] F. Iachello, Lie Algebras and Applications, Lect. Notes Phys. 708 (Springer, Berlin Heidelberg 2006), DOI 10.1007/b11785361

[17] N. A. Gromov and V. I. Manko, The Jordan-Schwinger representations of CayleyKlein groups. I. The orthogonal groups, J. Math. Phys. 31 (1990) 1047.

[18] N. A. Gromov and V. I. Manko, The Jordan-Schwinger representations of CayleyKlein groups. II. The unitary groups, J. Math. Phys. 31 (1990) 1054.

[19] N. A. Gromov and V. I. Manko, The Jordan-Schwinger representations of CayleyKlein groups. III. The symplectic groups, J. Math. Phys. 31 (1990) 1060.

[20] G. Mack and I.T. Todorov, Irreducibility of the ladder representations of U $(2,2)$ when restricted to the Poincare subgroup. J. Math. Phys. 10, 2078-2085 (1969)

[21] I.T. Todorov, Conformal Description of Spinning Particles, Trieste Notes in Physics, Springer-Verlag, Berlin (1986).

[22] I.T. Todorov, Discrete series of hermitean representations of the Lie algebra of U(p,q), International Centre for Theoretical Physics, Lecture Notes IC/66/71 (1966).

[23] R.L. Anderson, J. Fisher and R. Raczka, Coupling problem for $U(p, q)$ "ladder" representations, International Centre for Theoretical Physics, Lecture Notes IC/66/102 (1966).

[24] Y. Dothan, M. Gell-Mann and Y. Ne'eman, Series of hadron energy levels as representations of non-compact groups, Phys. Lett. 17, 148-151 (1965)

[25] G. Mack, All unitary ray representations of the conformal group $\mathrm{SU}(2,2)$ with positive energy, Comm. Math. Phys. 55 (1977) 1-28.

[26] W. Rühl, Distributions on Minkowski space and their connection with analytic representations of the conformal group, Commun. Math. Phys. 27 (1972) 53-86.

[27] W. Rühl, Field Representations of the Conformal Group with Continuous Mass Spectrum, Commun. Math. Phys. 30 (1973) 287-302. 
[28] M. Calixto and E. Pérez-Romero, Extended MacMahon-Schwinger's Master Theorem and Conformal Wavelets in Complex Minkowski Space, Appl. Comput. Harmon. Anal. 31 (2011) 143-168

[29] M. Calixto and E. Pérez-Romero, Conformal spinning quantum particles in complex Minkowski space as constrained nonlinear sigma models in $U(2,2)$ and Born's reciprocity, Int. J. Geom. Meth. Mod. Phys. 8 (2011) 587-619

[30] M. Calixto, E. Pérez-Romero and V. Aldaya, Coherent states of accelerated relativistic quantum particles, vacuum radiation and the spontaneous breakdown of the conformal $S U(2,2)$ symmetry, J. Phys. A: Math. Theor. 45 (2012) 244010

[31] H. Grosse, P. Presnajder and Z. Wang, Quantum field theory on quantized Bergman domain, J. Math. Phys. 53 (2012) 013508

[32] J.R. Klauder and Bo-Sture Skagerstam, Coherent States: Applications in Physics and Mathematical Physics, World Scientific (1985)

[33] A. Perelomov, Generalized Coherent States and Their Aplications, Springer-Verlag (1986)

[34] A. M. Perelomov, Coherent states for arbitrary Lie groups, Commun. Math. Phys. 26 (1972) 222-236

[35] S.T. Ali, J-P. Antoine, and J-P. Gazeau, Coherent States, Wavelets and Their Generalizations, Springer-Verlag, New York, Berlin, Heidelberg, 2000.

[36] J-P. Gazeau, Coherent States in Quantum Physics, Wiley-VCH, Berlin, 2009.

[37] R. Penrose, Twistor algebra, J. Math. Phys. 8 345-366 (1967)

[38] R. Penrose, Solutions of the Zero-Rest-Mass Equations, J. Math. Phys. 10 38-39 (1969)

[39] R. Penrose, M.A.H. MacCallum, Twistor Theory: an Approach to the Quantization of Fields in Space-Time, Phys. Rep. 6 (1972) 241-316

[40] R. Penrose, The twistor programme, Rep. on Math. Phys. 12 65-76 (1977)

[41] R. Penrose and W. Rindler, Spinors and Space-Time, Vols. 1 and 2, Cambridge University Press, Cambridge (1986).

[42] E. Witten, Perturbative Gauge Theory as a String Theory in Twistor Space, Commun. Math. Phys. 252 (2004) 189-258.

[43] A. Hodges, Theory with a twistor, Nature Physics 9 (2013) 205.

[44] A.A. Kirillov, Elements of the Theory of Representations, Springer, Berlin, (1976). 
[45] B. Kostant, Quantization and representation theory. In: Representation theory of Lie groups, London Mathematical Society. Lecture notes series 34, 91-150. Cambridge University Press 1979

[46] J.M. Souriau: Structure des systemes dynamiques, Dunod Paris (1970)

[47] W. Fulton and J. Harris, Representation Theory: a first course, Springer Verlag (New York) 1991

[48] H. D. Doebner, B. Gruber and M. Lorente, Boson operator realizations of $\operatorname{su}(2)$ and su(1,1) and unitarization, J. Math. Phys. 30 (1989) 594-600

[49] http://homepage.cem.itesm.mx/lgomez/quantum/ http://library.wolfram.com/infocenter/MathSource/4898/

[50] M. Calixto and E. Perez-Romero, Particle-hole entanglement, under investigation.

[51] R.S. Knox, Theory of Excitons, Academic Press Inc., New York and London (1963).

[52] S.A. Moskalenko and D.W. Snoke, Bose-Einstein condensation of excitons and biexcitons, Cambridge University Press, New York (2000)

[53] M. Calixto, Generalized $\mathcal{W}_{\infty}$ higher-spin algebras and symbolic calculus on flag manifolds, J. Geom. Phys. 56 (2006) 143-174

[54] A.J. Macfarlane, Generalizations of $\sigma$-models and $\mathbb{C} P^{N}$ models and instantons, Phys. Lett B 82 (1979) 239-241

[55] M. A. del Olmo, M. A. Rodríguez and P. Winternitz, The Conformal Group SU(2,2) and Integrable Systems on a Lorentzian Hyperboloid, Fortsch. Phys. 44 (1996) 199233.

[56] M. A. del Olmo, M. A. Rodríguez and P. Winternitz, Integrable systems based on SU(p,q) homogeneous manifolds, J. Math. Phys. 34 (1993) 5118.

[57] J. Dukelsky, S. Pittel and G. Sierra, Colloquium: Richardson-Gaudin models for many-body quantum systems, Rev. Mod. Phys. 76 (2004) 643-662

[58] G. Ortiz, R. Somma, J. Dukelsky, S. Rombouts, Exactly-solvable models derived from a generalized Gaudin algebra, Nucl. Phys. B 707 [FS] (2005) 421-457.

[59] M. Calixto and E. Perez-Romero, Coherent states on the Grassmannian $U(4) / U(2)^{2}$ : oscillator realization and bilayer fractional quantum Hall systems, J. Phys. A (Math. \& Theor.) 47 (2014) 115302 